\title{
Hybrid Broadband Ground-Motion Simulations: Combining Long-Period Deterministic Synthetics with High-Frequency Multiple $S$-to- $S$ Backscattering
}

\author{
by P. Martin Mai, Walter Imperatori, and Kim B. Olsen
}

\begin{abstract}
We present a new approach for computing broadband $(0-10 \mathrm{~Hz})$ synthetic seismograms by combining high-frequency (HF) scattering with low-frequency (LF) deterministic seismograms, considering finite-fault earthquake rupture models embedded in 3D earth structure. Site-specific HF-scattering Green's functions for a heterogeneous medium with uniformly distributed random isotropic scatterers are convolved with a source-time function that characterizes the temporal evolution of the rupture process. These scatterograms are then reconciled with the LF-deterministic waveforms using a frequency-domain optimization to match both amplitude and phase spectra around the target intersection frequency. The scattering parameters of the medium, scattering attenuation $\eta_{s}$, intrinsic attenuation $\eta_{i}$, and site-kappa, as well as frequency-dependent attenuation, determine waveform and spectral character of the HF-synthetics and thus affect the hybrid broadband seismograms. Applying our methodology to the 1994 Northridge earthquake and validating against near-field recordings at 24 sites, we find that our technique provides realistic broadband waveforms and consistently reproduces LF ground-motion intensities for two independent source descriptions. The least biased results, compared to recorded strong-motion data, are obtained after applying a frequency-dependent site-amplification factor to the broadband simulations. This innovative hybrid ground-motion simulation approach, applicable to any arbitrarily complex earthquake source model, is well suited for seismic hazard analysis and ground-motion estimation.
\end{abstract}

\section{Introduction}

Accurate ground-motion estimates for future earthquakes are needed by engineers to design buildings capable of withstanding the expected shaking levels. Standard practice estimates scalar ground-motion intensity measures (e.g., peak ground acceleration, PGA; peak ground velocity, PGV; spectral acceleration, $\mathrm{SA}_{T}$ at different periods $T$ ) using empirical ground-motion prediction equations, GMPEs (e.g., Abrahamson, 2006). However, ground-motion time series are needed as input for more realistic nonlinear structural dynamic analysis of buildings and performance-based earthquake engineering. Thus, modern earthquake engineering applications increasingly call for realistic simulations of near-source broadband ground motions to supplement strong-motion databases that suffer from limited near-source $(R \leq 10 \mathrm{~km})$ observations for large earthquakes. Realistic ground-motion synthetics may also help to verify (or even improve) GMPEs and reliably extend them into the distance range where only few recordings exist.

Conventional engineering approaches usually employ rather simplistic methods to generate ground-motion time series for building design. For example, two widely used methods simulate ground motions based on empiricalstochastic high-frequency approaches without considering potentially significant amplification effects from sedimentary basins (e.g., Boore, 1983; Beresnev and Atkinson, 1997). Moreover, these methods make strong simplifications regarding the kinematics of the earthquake source process, faultfiniteness, and three-dimensional (3D) wave-propagation effects. Another common method for generating time series for design purposes modifies and scales recorded near-field seismograms such that they match some target spectrum (Kramer, 1996). However, recent studies have demonstrated that such engineering practices may lead to biased estimates of the structural response (Bazzurro et al., 2004), indicating that these methods for simulating or rescaling ground-motions insufficiently capture the characteristics of real earthquake shaking.

Standard methods for ground-motion calculations due to earthquake rupture are generally designed to generate either the low-frequency (LF) or the high-frequency (HF) wave field 
but rarely both. For example, wavenumber integration (WI), finite difference (FD), or spectral element (SE) methods are often used to compute accurate LF ground motions in layered (WI) or 3D (FD, SE) Earth models. These calculations are typically carried out up to frequencies of $f \approx 1 \mathrm{~Hz}$ (depending on the lowest shear-wave velocity, the available computational resources, and the knowledge of Earth's structure). Because such synthetics are often not able to reproduce the observed ground-motion intensities at frequencies higher than about $0.5-1 \mathrm{~Hz}$, PGA values derived from LF wave-field simulations are inconsistent with empirical predictions. With the ever increasing availability of computational power, the lack of adequately resolved HF seismic energy is primarily due to imprecisely known Earth structure at short scale lengths where seismic scattering becomes important. However, incomplete knowledge about small-scale earthquake source dynamics and associated HF seismic radiation also contributes to the difficulty to compute a realistic broadband seismic wave field. In contrast, empirical-stochastic methods are designed to produce HF ground motions in agreement with data from historical earthquakes and GMPEs. These methods are often unable to match the LF waveforms, due to the use of simplified Earth models (hence neglecting the generation of large-amplitude surface waves) or the use of simplified source models in the far-field approximation (ignoring near-field effects and potential static displacements). Therefore, hybrid methods have been proposed to extend regional-scale LF wave-field calculations to frequencies above $f \approx 1 \mathrm{~Hz}$ for generating time series over the entire frequency range of engineering interest $(0 \leq f \leq 10 \mathrm{~Hz})$. In these methods, the LF and HF wave fields are computed separately (usually with different source descriptions for the LF and HF parts) and then combined to form broadband seismograms (e.g., Irikura, 1986; Berge et al., 1998; Kamae et al., 1998; Graves and Pitarka, 2004; Liu et al., 2006; Hartzell et al., 1999, 2005; Pulido and Kubo, 2004; Sørensen et al., 2007).

Broadband ground motions generated by such hybrid methods can be validated against empirically estimated ground-motion parameters (like PGA and $\mathrm{SA}_{T}$ ) using GMPEs but should also be calibrated against direct observational data (i.e., recordings of past earthquakes). It has been argued, however, that the structural response to such hybrid broadband ground motions may show unexpected behavior, in particular in the frequency range at which the two sets of seismograms are combined (Thrainsson et al., 2000). A possible explanation for the observed discrepancies is the inconsistency of the phase spectra of the two signals. While the amplitude spectra easily can be made to fit at the intersection frequency, the phase randomness of the HF signal often leads to a mismatch of LF and HF energy at the intersecting frequency and potentially to a biased structural response.

In this study, we propose an innovative method to compute realistic broadband near-source seismograms for engineering purposes, combining LF deterministic wave-field simulations with HF scattered waves. Our approach uses a
3D finite-difference method (Olsen, 1994) to calculate ground motions for complex source-rupture models embedded in a 3D velocity-density structure and contain all direct arrivals, converted phases, and surface waves, as well as the near-field terms radiated by the earthquake source. To compute the HF wave field, dominated by incoherently scattered seismic-wave energy, we calculate site-specific scattering Green's functions that are then convolved with a source-time function that encodes the temporal evolution of the earthquake rupture process. The resulting HF scattering seismograms are then combined with the LF finitedifference seismograms in the frequency domain such that both amplitude and phase spectra match optimally (Mai and Beroza, 2003).

In the following, we first present the theoretical framework for calculating the site-specific scattering operators, discuss the convolution with the source-time function to obtain site-specific seismograms, and explain the frequencydomain composition of broadband signals using HF and LF synthetics. In the second part of the study, we apply our method to compute strong ground-motion broadband synthetics for the 1994 M 6.7 Northridge event, a particularly well-recorded earthquake with near-field stations on both hard-rock and soft-soil sites. Moreover, the 3D velocity structure of the Los Angeles basin is relatively well known, making this event an ideal case for validating our method for hybrid broadband ground-motion simulation. Comparisons between data and simulations are carried out using scalar ground-motion intensities, PGA and PGV, as well as response spectral values in the period range $0.05 \leq T<10 \mathrm{~s}$, considering individual sites and ensemble statistics for a set of 24 strong-motion recording sites.

\section{Computation of Hybrid Broadband Seismograms}

For computing broadband hybrid seismograms, we adopt a three-stage approach. First, we calculate LF finitedifference synthetics for a finite-fault earthquake rupture embedded in a 3D Earth model. For simpler layered Earth models, computationally less expensive techniques can be used for calculating full wave-field synthetic seismograms. Second, we generate the HF scattering contributions for each observer location, considering path-averaged scattering properties and local site conditions based on site kappa $(\kappa)$. Finally, the two sets of seismograms are reconciled in the frequency domain to form hybrid broadband seismograms. Additional frequency-dependent site-effect corrections can be applied to the broadband synthetics, but we treat this part as a separate step in the procedure.

In the following, we briefly summarize multiple shearto-shear $(S$-to- $S)$ backscattering theory, which we use to compute the incoherent seismic wave field by generating site-specific backscattering Green's functions that capture the coda-wave characteristics. The HF-scattering seismogram is then obtained by applying a rupture-specific convolution operator (i.e., a properly scaled source-time function) to the 
scattering Green's function. The section closes with a short description on the combination of HF seismograms and LF seismograms that optimizes the matching of amplitude and phase spectra.

\section{Theory of Multiple $S$-to-S Scattering}

For calculating the site-specific scattering contribution, we apply the seismic-wave scattering theory for multiple $S$-to-S scattering (Zeng et al., 1991; Zeng, 1993). Starting from the general energy equation in a medium with unperturbed background velocity $v$ and random scatterers, Zeng et al. (1991) separate the incident wave-energy equation from the wave-energy equation for incoherently scattered waves. The scattering properties of the medium are characterized by uniformly distributed, random isotropic scatterers of density $n_{0}$. The scattering-wave energy can then be computed as the product of the scattering cross section $\sigma$, the wave-energy density at the scattering point, and the geometric spreading factor, as well as the energy redistribution due to elastic-wave scattering $\left(\eta_{s}\right)$ and the intrinsic attenuation due to energy dissipation $\left(\eta_{i}\right)$ occurring along the wave paths from the scattering point to the receiver. The total scattering coefficient $\left(\eta=\eta_{s}+\eta_{i}=n_{0} \cdot \sigma\right)$ is related to the extinction distance $L_{e}$ (e.g., Mayeda et al., 1992; Sato and Fehler, 1998), as $L_{e}=1 /\left(\eta_{s}+\eta_{i}\right) . L_{e}$ quantifies the distance at which the primary $S$-wave energy is decreased by $1 / e$. Hence, $\eta_{s}$ and $\eta_{i}$ have units of inverse distance. The scattering parameters can be recast in terms of quality factors, such that $Q_{s}^{-1}=\eta_{s} / k$ and $Q_{i}^{-1}=\eta_{i} / k$, where $k$ is the wavenumber.

Because the seismic scattering occurs within a volume $V$ that includes both the source and the site, the total scattering energy is determined by integrating the contributions from all scattering points in $V$. A compact integral solution for the scattered wave-energy equation has been derived by Zeng et al. (1991) for an unbounded 3D medium; their equation 24 quantifies the energy envelope of $S$-to- $S$ scattered waves as

$$
\begin{aligned}
E(\vec{r}, t)= & \frac{\delta\left(t-\frac{r}{v}\right) e^{-\eta v t}}{4 \pi v r^{2}}+\sum_{n=1}^{2} E_{n}(\vec{r}, t)+\left(\int_{-\infty}^{+\infty} \frac{e^{i \Omega}}{2 \pi} d \Omega\right. \\
& \left.\times \int_{0}^{\infty} \frac{\left(\frac{\eta_{s}}{k}\right)^{3} \times\left[\tan ^{-1}\left(\frac{k}{\eta+i \Omega / v}\right)\right]^{4} \times \sin (k r)}{2 \pi^{2} v r\left[1-\frac{\eta_{s}}{k} \tan ^{-1}\left(\frac{k}{\eta+i \Omega / v}\right)\right]} d k\right),
\end{aligned}
$$

where $r$ is distance, $t$ is time, and $v$ is the seismic velocity of the medium ( $i \Omega$ arises due to changing from the Laplace domain to the Fourier transform solution with respect to time; see Zeng et al. (1991) for details). The first term in equation 1 denotes the direct arrival, while the second term $\Sigma E_{n}$ captures the single-scattering energy $(n=1)$ and doublescattering $(n=2)$ energy; analytical solutions for $E_{1}(r, t)$ and $E_{2}(r, t)$ are given in Zeng et al., 1993, equations 21 and 22. The third contribution comprises the multiplescattered-wave energy in the medium. Equation 1 thus pro- vides the temporal energy decay of multiply isotropic backscattered waves in a uniform random medium for an impulsive source.

Because scattered waves arrive from all directions within the scattering volume $V$, Zeng et al. (1991) assumed isotropic radiation for direct waves, thus effectively removing radiation-pattern effects from the source. However, this can be accounted for by rescaling the energy equation (equation 1$)$ with an average radiation coefficient $(0.85)$, while a bounded medium can be considered by introducing a freesurface factor $(\sqrt{2}$; Zeng et al., 1995). Numerical experiments show that multiple backscattering becomes important as the scattering coefficient $\eta$ increases; the resulting energydecay curves will decrease more slowly, leading to longer and more pronounced coda-wave energy (Zeng et al., 1991). Note that equation 1 assumes $S$-to- $S$ scattering only and does not include surface-wave scattering.

For applying equation 1 to high-frequency groundmotion simulations, Zeng et al. (1995) propose a few modifications. While scattered waves arriving just prior to the $S$ wave are dominated by $S$-to- $P$ converted waves, which can be fully accounted for by computing the scattering conversion between $P$-waves and $S$-waves (Zeng, 1993), an approximation with single $S$-to- $P$ scattering conversion (Sato, 1977) is given as

$$
E_{s p}(\vec{r}, t)=\frac{\eta_{s p} e^{-\eta \beta t}}{4 \pi r \alpha t} \ln \frac{\vec{r} /(\beta t)+1}{\vec{r} /(\beta t)-1},
$$

where $\alpha$ and $\beta$ denote the $P$-wave and $S$-wave velocities, respectively (Zeng et al., 1995). For a Poisson solid, Zeng (1993) shows that $\eta_{s p}=\eta_{s}(\beta / \alpha)^{4} \approx 0.11 \eta_{s}$ and that the $P$-to- $S$ and $S$-to- $P$ scattering conversion energies behave as $E_{P-S}=E_{S-P}$. Moreover, because scattered $P$ waves after the $S$-wave arrival are rapidly converted into scattered $S$ waves, $S$-to- $S$ scattering will dominate (Aki, 1992). Hence, the wave field of scattered body waves is well approximated by multiple $S$-to- $S$ backscattering (Zeng et al., 1995). Equations 1 and 2 thus define the wave-energy decay with time, parameterizing the coda-wave envelope due to bodywave scattering. Surface-wave scattering is not explicitly incorporated into equation 1 or equation 2 , although it could potentially be important at distant sites due to the characteristic $1 / \sqrt{r}$ decay of surface-wave amplitudes. However, $S$-to- $S$ scattered wave energy will interact also with potentially occurring long-period surface waves; in such cases, we expect that the long-duration $S$-to- $S$ coda adequately approximates surface-wave scattering.

\section{Calculation of Site-Specific Scattering Operators}

For computing site-specific scattering contributions to the total wave field, we assume that scattering originates at the hypocenter of the earthquake, a simplification justified for small-to-moderate size earthquakes or, more generally, for the far-field regime. For large extended-fault ruptures 
and their associated near-field radiation due to localized slip patches, this approximation may not hold. The scattering contributions from such extended-source models and their effects on the broadband wave field are the subject of Mena et al. (2010).

To match the first-arrival times of the scattered highfrequency $P$ and $S$ waves with the arrival times of the low-frequency seismic wave field, we deploy a 3D finitedifference ray-tracing method (Hole, 1992) for computing phase arrival times. This step also ensures adequate performance of the frequency-domain wave-field composition, optimizing both amplitude and phase matching of the HF motions and LF motions (Mai and Beroza, 2003).

To constrain the scattering parameters in equation 1 , $\eta_{s}$ and $\eta_{i}$, we begin with Zeng et al. (1991), who used generic values of $\eta_{i}=0.01 \mathrm{~km}^{-1}$ and $\eta_{s}$ in the range 0.002 to $0.05 \mathrm{~km}^{-1}$. They showed how multiple scattering becomes important as the scattering coefficient increases. Alternatively, the dimensionless seismic albedo, $B_{0}=\frac{\eta_{s}}{\eta_{s}+\eta_{i}}$, can be used to quantify scattering parameters. $B_{0}$ is directly related to the extinction distance, $L_{e}$ (e.g., Mayeda et al., 1992). These authors estimate frequency-dependent $S$-wave scattering parameters and absorption coefficients in central California, reporting values for $B_{0}$ in the range of $0.26-$ 0.78 , leading to extinction distances (scattered waveamplitude decrease by a factor of $1 / e$ ) of about $L_{e}=$ 20-30 km (Mayeda et al., 1992). Based on these measurements, Mayeda et al. (1992) calculate the scattering attenuation $\eta_{s}$ to $0.033,0.015,0.066$, and $0.046 \mathrm{~km}^{-1}$ for frequencies of $1.5,3.0,6$, and $9 \mathrm{~Hz}$, respectively (assuming a shear-wave speed of $\beta=3.7 \mathrm{~km} / \mathrm{s}$ ), while intrinsic attenuation $\eta_{i}$ ranges from 0.018 to $0.032 \mathrm{~km}^{-1}$. In a case study of HF groundmotion simulation for the 1991 Uttarkashi (India) earthquake, Zeng et al. (1995) adopt $B_{0}=0.4, \eta_{s}=0.025 \mathrm{~km}^{-1}$, and $\eta_{i}=0.0125 \mathrm{~km}^{-1}$. For the purpose of our methodological study, we fix the scattering parameters to $\eta_{i}=0.028$ and $\eta_{s}=0.01$; however, we note that $\pm 20 \%$ variation in these values does not change the peak motions noticeably. A detailed parameter study on the effects of these scattering parameters on several strong-motion parameters is reported in the companion paper by Mena et al. (2010).

When computing the site-specific seismic scattering, site kappa $\kappa$ is used to characterize the shape of the acceleration spectra at high frequencies as $A(f)=A_{0} \cdot \exp (-\pi \kappa f)$ (Hough and Anderson, 1988). Site kappa thus controls the $\mathrm{HF}$ amplitudes; that is, increasing $\kappa$ decreases the HF seismic energy. Values for $\kappa$ can be obtained from empirical relations (e.g., Hough and Anderson, 1988), or by adopting the conjecture of Abercrombie (1997) that $90 \%$ of the total seismic attenuation in bedrock occurs in the upper $3 \mathrm{~km}$ of the Earth's crust. Because $\kappa$ is defined as $\frac{R}{Q_{s} v_{s}}(R$ is relevant path-length of the attenuated seismic wave, $3 \mathrm{~km}$ in this case; $Q_{s}$ and $v_{s}$ are $S$-wave quality factor and speed, respectively). $\kappa$ can then be computed for known velocity-attenuation structure. For the purpose of this work, we initially fix site kappa at $\kappa=0.03$, a value close to the estimate for generic rocks $(\kappa=0.035-0.04$; Boore and Joyner, 1997); subsequently, we estimate $\kappa$ from the strong-motion recordings.

To complete the characterization of the scattering operator, frequency-dependent path attenuation is applied as $Q(f)=Q_{0} \times f^{n}$. A number of studies suggest different values for $Q_{0}$ and $n$ (e.g., $Q_{0}=180, n=0.45$ by Raoof $e t$ al., 1999; $Q_{0}=150, n=0.60$ by Atkinson and Silva, 1997; $Q_{0}=400, n=0.60$ by Boatwright et al., 2002). Based on these findings, we adopt the $Q$ model $Q(f)=150 \times$ $f^{0.6}$ for our calculations.

To generate the site-specific scattering Green's function, we use equation 1 to compute the temporal energy decay of multiply-scattered waves in a uniform random isotropic medium for an impulsive source. Equation 1 defines the envelope of a scattered seismic signal. Assuming a large number of elementary scatterers $\left(N \approx 10^{2}-10^{4}\right)$ distributed uniformly throughout the scattering volume $V$, each acting as local pointlike scatterer, we generate random events of uniformly distributed amplitude between $\pm \sqrt{3}$, assuring a mean square wave amplitude (energy) of unity (Zeng et al., 1995). These events are then shaped by the envelope of scattered-wave energy (equation 1). This procedure also accounts for the $P$ and $S$ arrival times, calculated for each site by a 3D ray-tracing method.

The scattering Green's function is calculated for a source strength equivalent to $1 \mathrm{~cm}$ of displacement occurring on a rectangular fault patch of size $1 \times 1 \mathrm{~km}^{2}$. For typical crustal rocks with shear modulus $\mu=35 \mathrm{GPa}$, this translates to a seismic moment of $M_{0}=3.5 \times 10^{14} \mathrm{Nm}\left(M_{\mathrm{w}} 3.66\right)$, implying a static stress drop of $0.35 \mathrm{MPa}$ using the standard expression for static stress drop $\Delta \sigma=C \cdot \mu \frac{D}{L_{c}}$ (where $D$ is the mean slip, $L_{c}$ a characteristic length scale of the fault, and $C$ a constant of order unity that depends on faulting style and geometry). To apply the signature of the source mechanism and to scale the HF-frequency contribution to the correct amplitude level for the target source strength, the scattering Green's function needs to be convolved with an appropriate source wavelet. Zeng (1993) proposes a generic wavelet of the form $f(t)=\gamma \sqrt{2} \exp \left(-\gamma^{2} t^{2}\right) \quad(\gamma=0.3)$. However, we are interested in simulating the HF contributions due to earthquake rupture; we thus parameterize the source wavelet based on the temporal rupture evolution. For this reason, we define the source wavelet (i.e., the convolution operator applied to the scattering Green's function) in terms of the earthquake's source-time function (STF). This source-time function can either be a generic (theoretical) slip-rate function (e.g., boxcar, isosceles triangle, Kostrovlike) scaled to the target seismic moment (see accompanying paper by Mena et al., 2010, for details) or can be directly computed from the selected finite-fault rupture model.

The convolution with an appropriate STF scales the scattering Green's functions to the target seismic moment and introduces the long-period signature of the spatiotemporal rupture evolution. We denote the resulting HF seismograms as scatterograms to indicate that they represent a realization 
of the HF content of the seismic wave field based on multiple $S$-to- $S$ backscattering theory. It is important to note that the convolution with the STF also ensures the appropriate spectral properties of the scatterograms. While the scattering Green's function exhibits a white noise spectrum, the velocity spectrum of the scatterogram obtains a $1 / f$ decay through the STF convolution operator. This spectral behavior of the HF scatterogram is needed for rendering it suitable to compute hybrid broadband seismograms. Figure 1 displays an example of the scattering Green's function (Fig. 1a), the scatterogram after convolving the scattering Green's function with an appropriate STF (Fig. 1b) and the corresponding amplitude spectrum (Fig. 1c).

Combining Low-Frequency and

High-Frequency Seismograms

Several methods have been proposed for combining HF and LF seismograms to compute hybrid broadband ground- motions (Irikura and Kamae, 1994; Hartzell et al., 1999; Berge et al., 1998; Graves and Pitarka, 2004; Pulido and Kubo, 2004; Liu et al., 2006). These techniques use different parameterizations for the earthquake rupture process, for computing the LF and HF wave field, and for combining the two sets of seismograms. The latter step can be carried out either in the time domain (e.g., Berge et al., 1998; Graves and Pitarka, 2004) under consideration of the appropriate amplitude-scaling of the two time series or in the frequency domain (Hartzell et al., 1999; Pulido and Kubo, 2004) by applying a pair of matched filters. In almost all cases, the HF wave field is computed as a stochastic time series following the approach of Boore (1983), with fixed matching frequency near $1 \mathrm{~Hz}$, based on the seismological observation that source radiation and wave-propagation effects tend to become random at frequencies above $f \approx 1 \mathrm{~Hz}$ (e.g., Pulido and Kubo, 2004). However, the transition from the theoretical radiation pattern of a double-couple source to isotropic radiation may not be abrupt. Based on observational
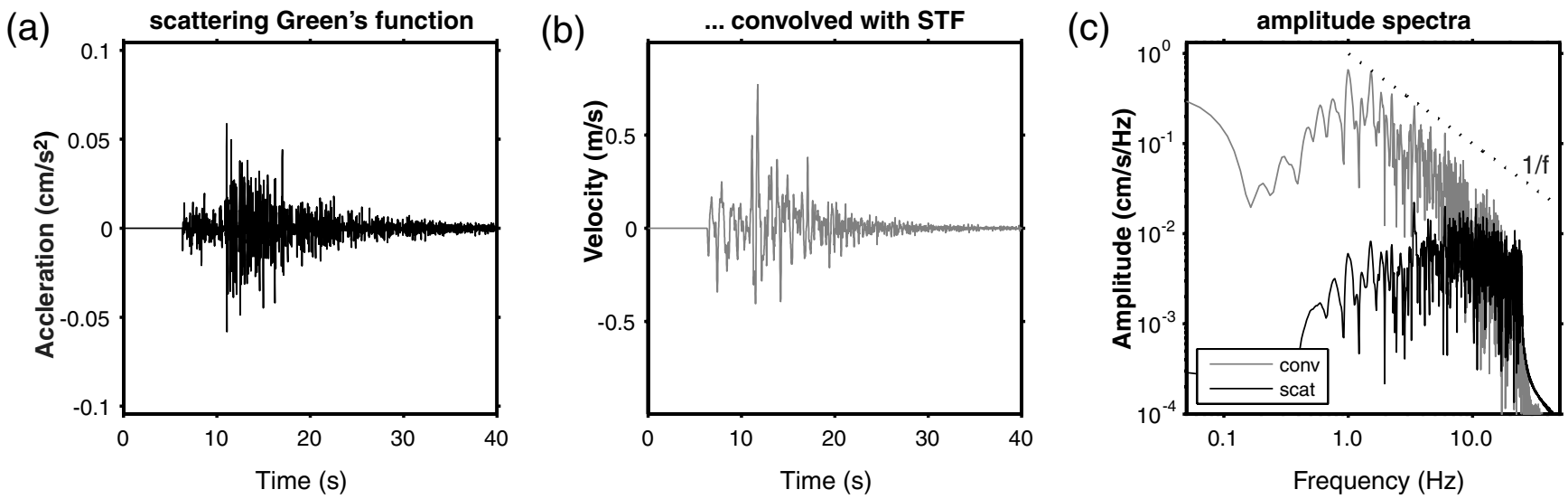

hybrid broadband seismogram composition

(d)

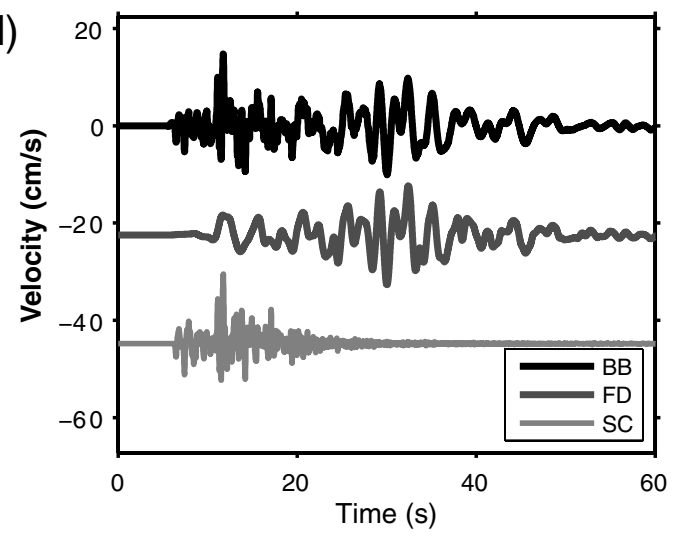

(e)

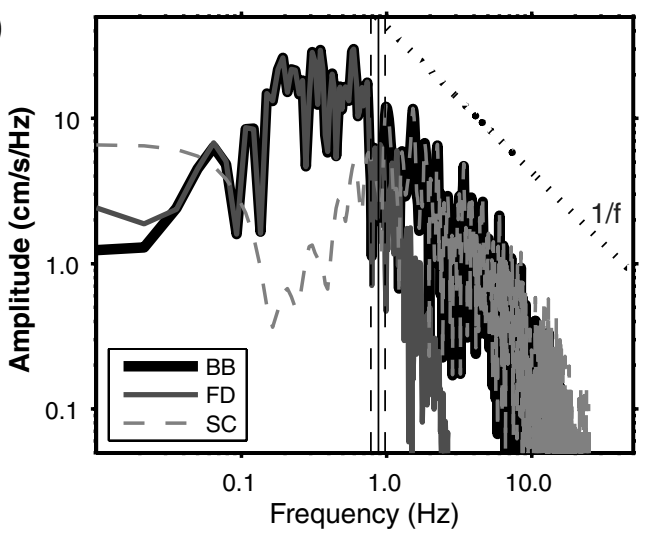

Figure 1. Computation of hybrid broadband seismograms using scattering Green's functions. (a) Site-specific scattering Green's function for a point-source at the hypocenter; (b) scatterogram, formed by convolving the scattering Green's function in (a) with a source-time function (STF) representing the temporal rupture evolution. (c) Fourier amplitude spectra for the time series in (a) and (b); the velocity-scatterogram decays as $1 / f$ (dotted line) beyond the corner frequency; (d) broadband seismogram (top) computed by combining the LF seismogram (center) with the site-specific HF scatterogram (bottom) using a Fourier-domain amplitude-and-phase matching technique (Mai and Beroza, 2003). (e) Amplitude spectra for the time series in (d); the spectra of the broadband synthetics represent the LF motions at low frequencies (except for a small shift at the lowest frequency due to filtering of the LF synthetics) and the HF-scattering contribution at high frequencies (matching frequency and search range shown by vertical lines). 
evidence (Liu and Helmberger, 1985; Satoh, 2002; Takenaka et al., 2003). Pulido and Kubo (2004) suggest that this transition occurs in the frequency range between 1 and $4 \mathrm{~Hz}$, in which the radiation pattern gradually changes from doublecouple to isotropic radiation. It is therefore expected that directivity effects (due to rupture propagation toward or away from a site) will strongly decrease and eventually disappear in this frequency range as scattered wave energy becomes dominant. This conjecture is in agreement with recent observational and numerical evidence (Boatwright et al., 2002; Watson-Lamprey, 2007; Spudich and Chiou, 2008; Gritz, 2009).

The previously mentioned frequency-domain matching techniques consider only the amplitude spectra of the two time series and neglect the phase information for creating broadband synthetics. However, engineering investigations on such hybrid waveforms have shown that the structural response of such synthetics is often unrealistic and inconsistent with the response of observed records at the matching frequency (Bazzurro et al., 2004). A possible explanation for this observation is that matching only the amplitude spectra insufficiently captures the spectral energy levels because the HF and LF phases are not synchronized at the intersection frequency.

The spectral-matching approach proposed by Mai and Beroza (2003) attempts to avoid this spectral energy leakage at the intersecting frequency by simultaneously optimizing the amplitude and phase matching within a small frequency band around the target intersection frequency. This method assumes that the high-frequency seismograms are not entirely stochastic time series but instead contain the correct primary phase arrivals of the direct $P$-waves and $S$-waves, as well as some signature of the earthquake rupture process. In this study, we compute the HF-scattered wave field based on HF coda waves with consistent primary phase-arrival times (obtained from 3D ray tracing), modulated by a convolution operator to apply an earthquake source-time function to the HF-scattering seismograms. Thus, our scatterograms satisfy the requirements of the Mai and Beroza (2003) approach, and we deploy their technique to calculate broadband synthetic seismograms. The spectral addition of the HF scatterogram and the LF time series for generating the final broadband seismogram is depicted in Figure 1d,e. The target intersection frequency in this case is $f_{m}=0.8 \mathrm{~Hz}$, and the algorithm searches within a small frequency band $\left(f_{m} \pm\right.$ $0.1 \mathrm{~Hz}$ ) for the best-matching amplitude and phase (Fig. 1e). The matching frequency $f_{m}$ is chosen such that the reliably computed frequency content of the LF-wave field is utilized as much as possible. In the present example, the highest resolved frequency in the 3D finite-difference calculations is $f \approx 1 \mathrm{~Hz}$, therefore we set $f_{m}=0.8 \pm 0.1$. The matching frequency is thus chosen to be within the range in which the transitions from deterministic double-couple radiation to stochastic isotropic radiation occurs.

To achieve continuity in the Fourier amplitude spectra at the intersecting frequency, an additional small spectralamplitude adjustment is needed for the HF waveforms. This adjustment is obtained by computing the average ratio of the LF and HF spectral amplitude in the range of the matching frequency, and then scaling the HF spectra such that the two spectral levels are equal at the matching frequency $f_{m}$. Because the scatterograms are already scaled to the target seismic moment, these adjustments are generally small, in the range of a factor of 0.5-2.0. Albeit small, this spectral scaling ensures that artificial spectral steps in the broadband synthetics are minimized.

\section{Broadband Simulations for the 1994 Northridge Earthquake}

We apply our broadband ground-motion simulation methodology to the 1994 M 6.7 Northridge (California) earthquake and compare hybrid synthetics to strong-motion recordings at 24 sites (Fig. 2). The Northridge earthquake was selected for validation of our method because of its large strong-motion data set. Moreover, the event provides an ideal test case for our method because several earthquake rupture models are available and because the 3D-velocity structure of the Los Angeles basin is relatively well known. In the following sections, we explain our parameter choices and the particular modeling setup; we then examine our simulation results at two sites in detail before providing ensemble statistics to characterize the overall performance of our methodology.

We generated the LF synthetics using a 3D-finitedifference method in the frequency range $0-1.0 \mathrm{~Hz}$ (Olsen, 1994) with a minimum shear-wave velocity of $1 \mathrm{~km} / \mathrm{s}$ and a constant grid spacing of $d x=200 \mathrm{~m}$ using the Southern California Earthquake Center (SCEC) Community Velocity Model (CVM 3.0, Kohler et al., 2003). The FD code solves the $3 \mathrm{D}$ velocity-stress wave equation explicitly by a staggered-grid FD method, fourth-order accurate in space and second-order accurate in time, and includes a coarsegrained implementation of the memory variables for a constant- $Q$ solid (Day, 1998; Day and Bradley, 2001). The $Q$ values in the 3D velocity-density model are based on $Q$ relations validated against data for the Northridge earthquake (Olsen et al., 2003). Perfectly matched layers (Marcinkovich and Olsen, 2003) were used to implement absorbing boundary conditions on the sides and bottom of the grid, and a zerostress free-surface boundary condition was used at the top (Gottschämmer and Olsen, 2001). The code has been extensively validated for a wide range of problems, from simple point-sources in a half-space to complex ruptures on dipping faults in 3D crustal models (Day et al., 2008). We selected 24 strong-motion sites that recorded the Northridge earthquake, with hypocentral distances $\left(R_{\mathrm{hyp}}\right)$ in the range of $18-37 \mathrm{~km}$ (closest distance to the fault plane $R_{\mathrm{flt}}=5-31 \mathrm{~km}$ ) and even azimuthal distribution (Fig. 2, Table 1).

\section{Earthquake Source Parameters}

For the 1994 Northridge earthquake, a number of source-inversion results have been published in the past, 


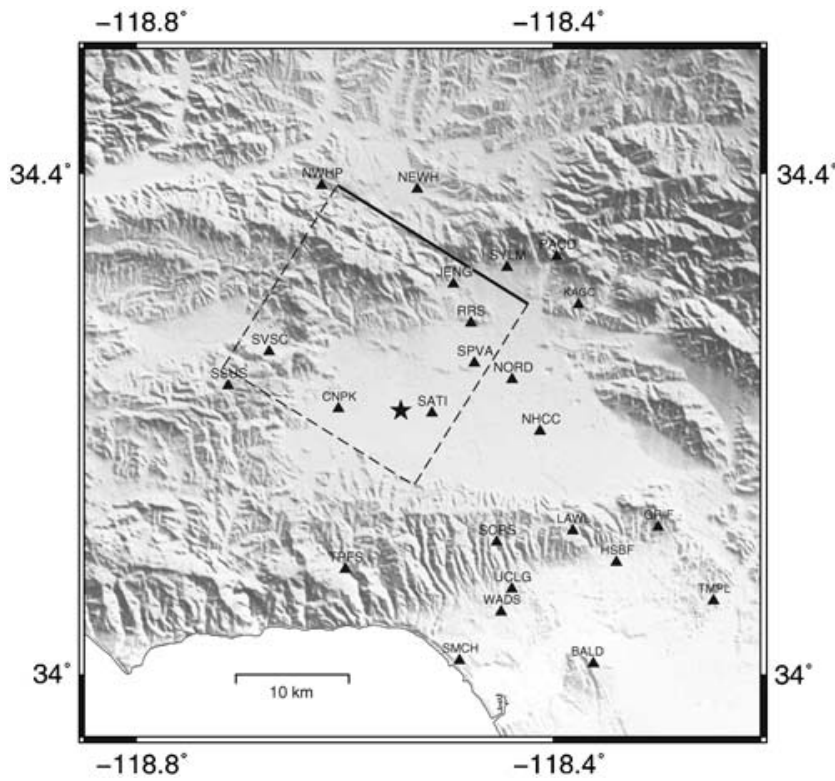

Figure 2. Map showing the locations for a set of 24 stations in the Los Angeles area that recorded the M 6.7 Northridge earthquake and for which we simulated broadband ground motions. The star marks the epicenter; the dotted line shows the surface projection of the rupture plane (strike $\Phi=122^{\circ}, \operatorname{dip} \delta=40^{\circ}$ toward the southwest; top of the fault plane [thick black line] at 5-km depth).

giving us the opportunity to test the sensitivity of our approach to the earthquake rupture process. For our analysis, we select two source models derived in independent source- inversion studies: the slip models of Wald et al. (1996) and Hartzell et al. (1996) (Fig. 3 and Fig. 4, hereafter referred to as the Wald model and the Hartzell model, respectively). Both source models are characterized by heterogeneous slip and variable rake angle and have been obtained by inverting near-source strong-motion recordings (Wald et al., 1996, augmented their analysis with teleseismic data and geodetic measurements). Using the slip distribution of the Wald model, we generate the spatiotemporal rupture process based on the pseudodynamic source characterization (Guatteri et al., 2004) such that the variability in rise time and rupture speed on the fault is consistent with first-order principles of earthquake source dynamics (i.e., rise time approximately follows a crack model with longer rise times at the hypocenter; rupture speed increases over regions with higher stress/ slip). For computing the LF finite-difference synthetics for the Wald model, we assume an isosceles triangle as the slip-velocity function. The Hartzell model has been generated as part of the SCEC Broadband Ground-Motion Simulation Project (Robert Graves, personal comm.) based on the published final slip distribution (Hartzell et al., 1996). The variability in the temporal rupture evolution is based on a set of empirical rules that link changes in rupture speed and rise time to the underlying slip distribution: shorter rise times and faster rupture propagation occur over regions of high slip (Graves and Pitarka, 2010). To calculate the LF-seismograms for the Hartzell model, the composite slip-velocity function of Liu et al. (2006) is used.

Table 1

Station Information for a Subset of 24 sites that Recorded the 1994 M 6.7 Northridge Earthquake*

\begin{tabular}{clrrrrrrrr}
\hline Number & Station Name & Longitude $\left(^{\circ}\right)$ & Latitude $\left({ }^{\circ}\right)$ & $R_{\text {hyp }}(\mathrm{km})$ & $R_{\mathrm{flt}}(\mathrm{km})$ & $f_{\min }(\mathrm{Hz})$ & $f_{\max }(\mathrm{Hz})$ & $\kappa(\mathrm{s})$ & $V_{S 30}(\mathrm{~m} / \mathrm{s})$ \\
\hline 1 & BALD & -118.3611 & 34.0087 & 33.2 & 29.9 & 0.160 & 23 & 0.045 & 297 \\
2 & CNPK & -118.6060 & 34.2126 & 18.2 & 14.7 & 0.050 & 30 & 0.030 & 268 \\
3 & GRIF & -118.2987 & 34.1180 & 30.9 & 23.8 & 0.083 & 50 & 0.035 & 1016 \\
4 & HSBF & -118.3390 & 34.0897 & 29.4 & 24.0 & 0.200 & 23 & 0.040 & 317 \\
5 & JENG & -118.4955 & 34.3120 & 21.8 & 5.4 & 0.083 & 50 & 0.010 & 373 \\
6 & KAGC & -118.3752 & 34.2956 & 26.0 & 7.3 & 0.140 & 23 & 0.045 & 508 \\
7 & LAWL & -118.3807 & 34.1150 & 25.8 & 20.3 & 0.114 & 30 & 0.025 & 1223 \\
8 & NEWH & -118.5302 & 34.3876 & 26.8 & 5.9 & 0.120 & 23 & 0.025 & 269 \\
9 & NHCC & -118.4123 & 34.1945 & 21.9 & 12.5 & 0.100 & 30 & 0.015 & 446 \\
10 & NORD & -118.4390 & 34.2358 & 20.7 & 8.7 & 0.120 & 23 & 0.010 & 298 \\
11 & NWHP & -118.6221 & 34.3907 & 27.8 & 5.5 & 0.071 & 30 & 0.035 & 286 \\
12 & PACD & -118.3961 & 34.3339 & 26.9 & 7.0 & 0.160 & 23 & 0.050 & 2016 \\
13 & RRS & -118.4787 & 34.2810 & 20.6 & 6.5 & 0.073 & 30 & 0.030 & 282 \\
14 & SATI & -118.5162 & 34.2091 & 17.8 & 12.1 & 0.100 & 30 & 0.060 & 281 \\
15 & SMCH & -118.4898 & 34.0111 & 28.5 & 26.5 & 0.140 & 23 & 0.065 & 336 \\
16 & SPVA & -118.4754 & 34.2490 & 19.5 & 8.4 & 0.083 & 50 & 0.025 & 380 \\
17 & SSUS & -118.7120 & 34.2308 & 22.8 & 16.7 & 0.083 & 50 & 0.010 & 715 \\
18 & SYLM & -118.4440 & 34.3254 & 24.2 & 5.3 & 0.120 & 23 & 0.040 & 441 \\
19 & TMPL & -118.2455 & 34.0590 & 37.1 & 31.5 & 0.200 & 46 & 0.010 & 376 \\
20 & TPFS & -118.5995 & 34.0841 & 22.5 & 22.3 & 0.083 & 50 & 0.010 & 376 \\
21 & UCLG & -118.4393 & 34.0684 & 25.6 & 22.5 & 0.080 & 25 & 0.010 & 316 \\
22 & WADS & -118.4498 & 34.0500 & 26.2 & 23.6 & 0.083 & 50 & 0.025 & 392 \\
23 & SCRS & -118.4540 & 34.1060 & 22.7 & 19.1 & 0.300 & 23 & 0.050 & 438 \\
24 & SVSC & -118.6660 & 34.2640 & 21.3 & 13.4 & 0.625 & 30 & 0.065 & 557 \\
\hline
\end{tabular}

* Geographical coordinates, hypocentral distance $\left(R_{\mathrm{hyp}}\right)$, closest distance to the fault $\left(R_{\mathrm{flt}}\right)$, usable frequency range $\left(f_{\min }, f_{\max }\right)$ according to the Next Generation Attenuation (NGA) data file, estimated site kappa (this study), and $V_{S 30}$ according to the NGA data file. 
(a)

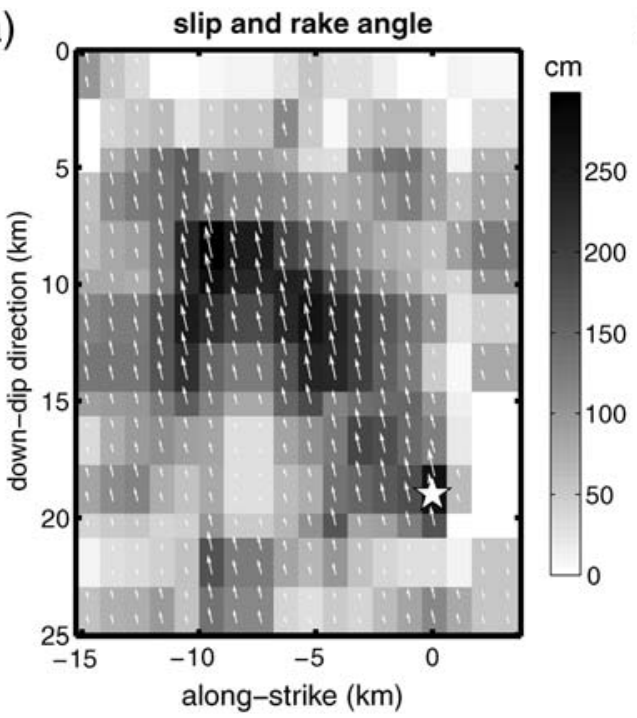

(b) rupture-time contours and rise time

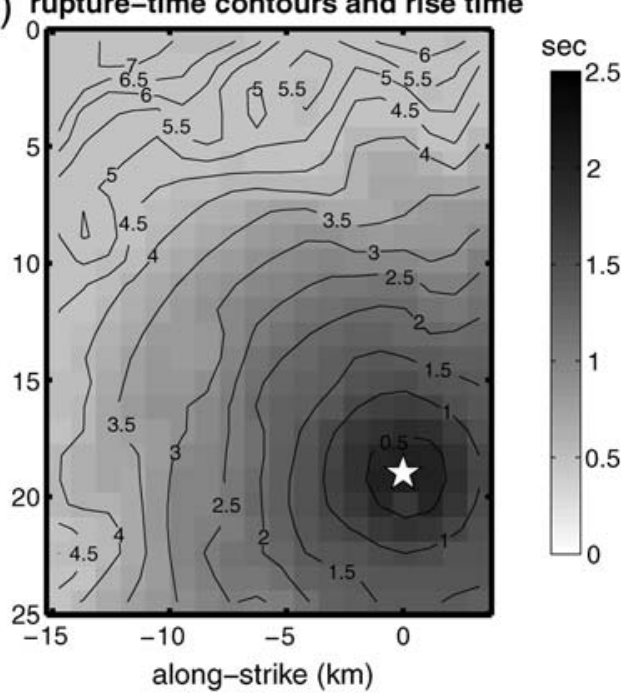

(c)

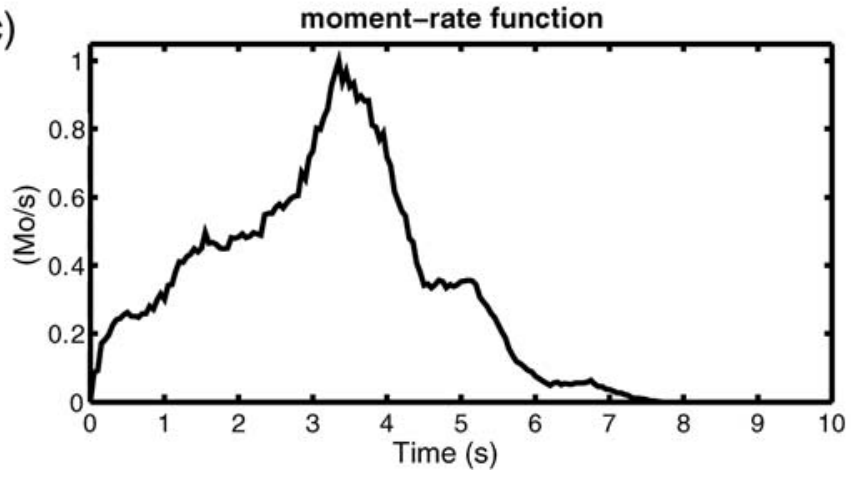

Figure 3. Rupture model for the 1994 Northridge earthquake based on the slip inversion of Wald et al. (1996). The temporal rupture evolution (rise time, rupture onset times) is computed using the pseudodynamic approach of Guatteri et al. (2004). (a) Gray-shaded slip amplitudes (in $\mathrm{cm}$ ), with white arrows indicating the rake angle; the white star marks the hypocenter. (b) contoured rupture-front propagation times on top of the gray-shaded rise time distribution. (c) Normalized moment-rate function computed from the rupture model (see text for details).

These two rupture models with variable slip, rise time, and rupture speed are used to compute the corresponding source-time function for convolution with the scattering Green's function. The bottom panels in Figure 3 and Figure 4 display the (normalized) moment-rate function, calculated by time-integrating the seismic moment released along each rupture-time contour, accounting also for the corresponding slip-velocity function used in the particular model. Thus, the two resulting source-time functions reflect the differences in the spatiotemporal rupture evolution of the two models. In the case of the Wald model, the STF is dominated by a single peak at about $4 \mathrm{~s}$ when the rupture front reaches the large slip asperity at down-dip distance $z \approx 8 \mathrm{~km}$. The Hartzell model is characterized by multiple asperities rupturing at distinctively different times, resulting in an STF with three peaks of different amplitude.

The two source models depicted in Figure 3 and Figure 4 not only differ in their temporal rupture evolution but also in their final seismic moment: $M_{0} 1.75 \times 10^{19} \mathrm{Nm}\left(M_{\mathrm{w}} 6.79\right)$ and $M_{0} 1.02 \times 10^{19} \mathrm{Nm}\left(M_{\mathrm{w}} 6.64\right)$ for the Wald model and the Hartzell model, respectively. Note that these estimates do not exactly reflect the moment estimates published in the literature because the rupture models have been slightly modified. Using Ripperger and Mai (2004) to compute the associated static stress drop, we obtain average stress drops of $\Delta \sigma=3-4 \mathrm{MPa}$ when considering the entire fault plane (which includes substantial regions of low slip and hence negative stress drop) and values of $\Delta \sigma=10-12 \mathrm{MPa}$ when considering only the fault regions with positive stress drop. However, the local stress drop on the large-slip asperities is higher, reaching 15-19 $\mathrm{MPa}$ on the deeper slip patch (estimates vary depending on particular source model and the area over which the stress drop is averaged) and 11-14 MPa on the shallow slip patch. Corresponding maximum stress drops are on the order of 37-54 MPa and 17-30 MPa for the deep and shallow asperity, respectively.

These stress-drop estimates are important because they define the factor by which the STF of the rupture is scaled before being applied to the scattering Green's function, for which the stress drop is about $0.35 \mathrm{MPa}$ (see previous 
(a)

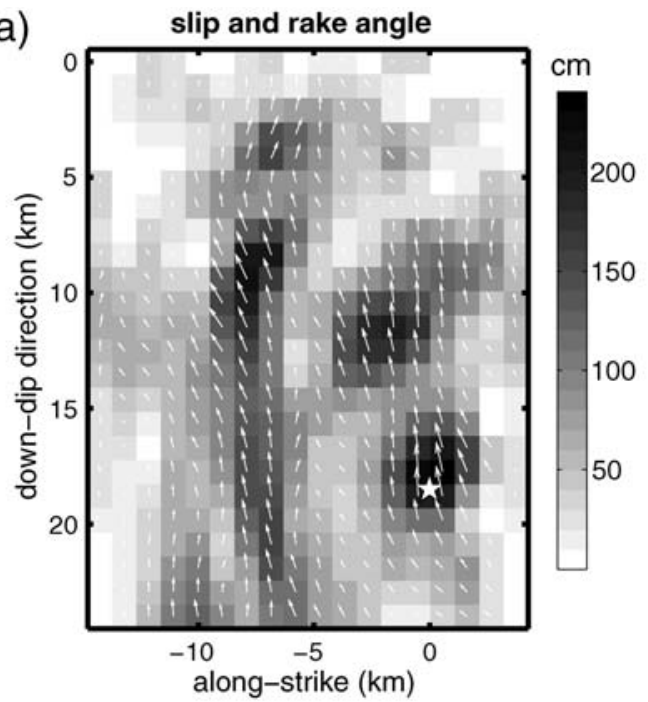

(b) rupture-time contours and rise time

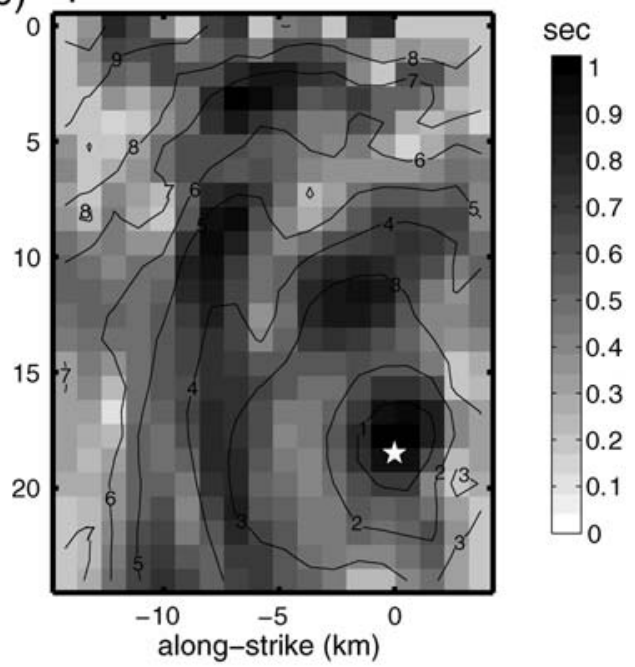

(c)

moment-rate function

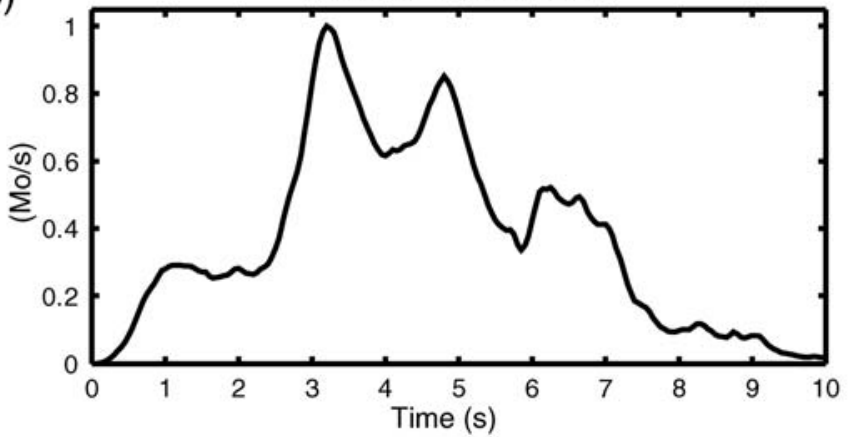

Figure 4. Rupture model for the 1994 Northridge earthquake based on the slip inversion of Hartzell et al. (1996), with stochastic component added to the slip and rupture speed to ensure sufficient high-frequency radiation from the source (Robert Graves, personal comm.). The temporal rupture evolution (rise time, rupture onset times) was provided also by R. Graves. (For additional details, see also caption to Fig. 3).

description). Because we use the hypocenter as the origin for computing the scattering Green's function (and associated seismic phase arrivals), the final scale factor is determined by the stress drop on the deep large-slip asperity located at the hypocenter.

Given these stress-drop estimates and the seismic moment of the two rupture models, we compute the corner frequency $f_{c}$ of the earthquake to anticipate the spectral properties of the seismograms. Following Allmann and Shearer (2009), the stress drop $\Delta \sigma$ for a circular rupture with rupture speed $v_{r}=0.9 \beta$ (assuming shear-wave speed $\beta=3.9 \mathrm{~km} / \mathrm{s})$ is given as $\Delta \sigma=M_{0}\left(\frac{f_{c}}{0.42 \beta}\right)^{3}$, from which we obtain $f_{c}=0.10 \mathrm{~Hz}$ for the fault-averaged stress drop $\Delta \sigma=4 \mathrm{MPa}$ and the lower moment estimate, and $f_{c}=0.22 \mathrm{~Hz}$ assuming the maximum stress drop on the fault $(\Delta \sigma=54 \mathrm{MPa})$ and larger magnitude. Although these are first-order estimates of the corner frequency, we concludes that our 3D finite-difference simulations (maximum resolved frequency $\approx 1 \mathrm{~Hz}$ ) reach well beyond the corner frequency into the decaying part of the spectra. The strongmotion data support this corner-frequency estimate (see
Fig. 5 and Fig. 6), although the amplitude spectra show more complexities at higher frequencies. Note that our choice for the matching frequency of $f_{m}=0.8 \mathrm{~Hz}$ is well beyond the corner frequency.

\section{Near-Field and Far-Field Effects}

It is important to consider the frequency-distance ranges in which near-field and far-field terms dominate. Ichinose et al. (2000) discuss criteria for the relative importance of near-field, intermediate-field, and far-field terms in layered Earth models. Although the geologic conditions are more complex in the Los Angeles basin, their analysis provides a first-order estimate of the frequencies at which near-field terms should be important, based on the source-site geometry for this earthquake. According to the condition $\frac{\omega \times R}{c} \ll 1$, where $\omega$ is angular frequency, $R$ is hypocentral distance, and $c$ is the respective wave speed, the near-field is significant for all $R$ within a small fraction of wavelength $\gamma$ from the source. Hence, near-field terms may be important out to regional distances $(R=200-500 \mathrm{~km})$ for low frequencies 
East-West Component $(\mathrm{cm} / \mathrm{s})$

(a)

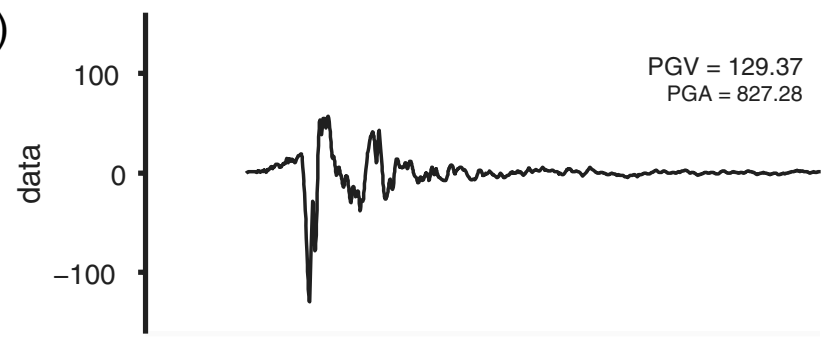

(c)

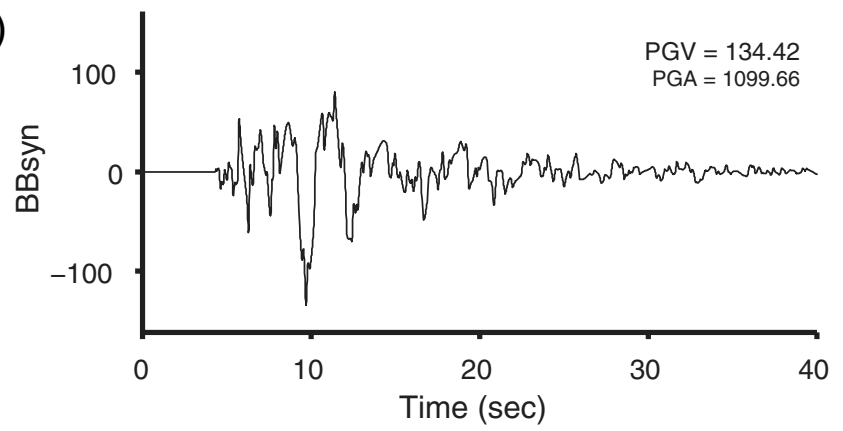

(e)

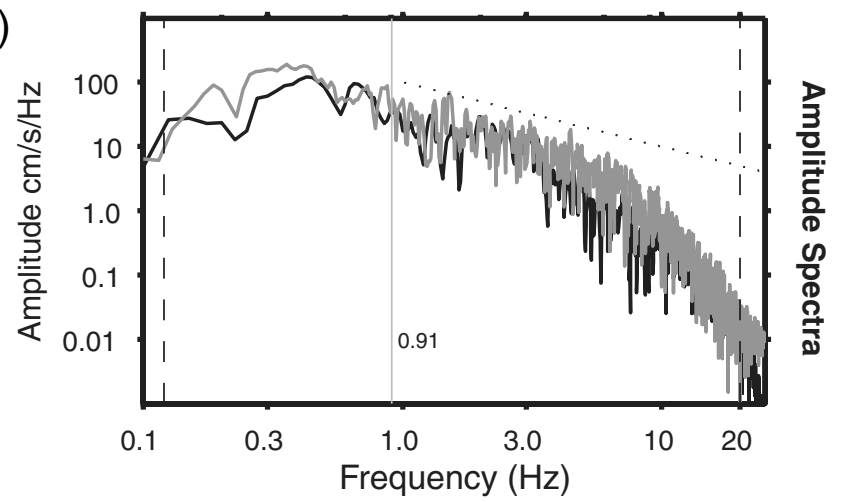

(g)

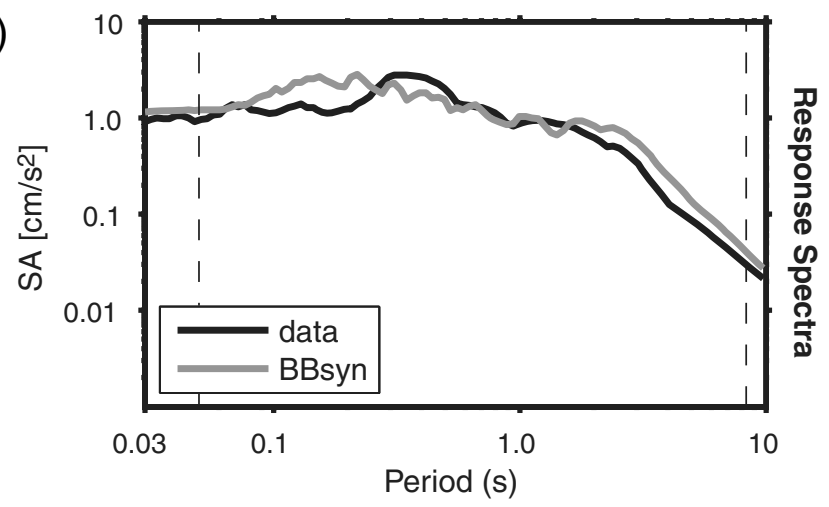

North-South Component $(\mathrm{cm} / \mathrm{s})$

(b)

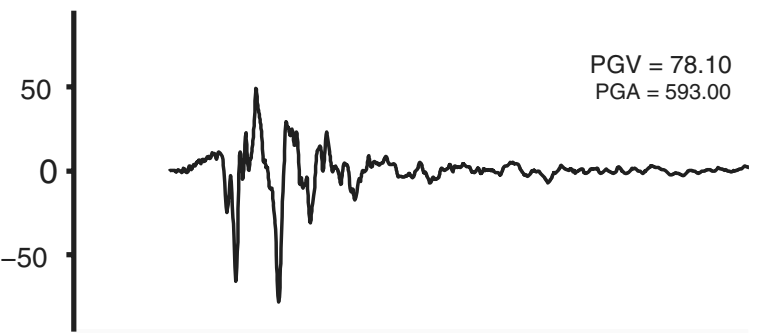

(d)

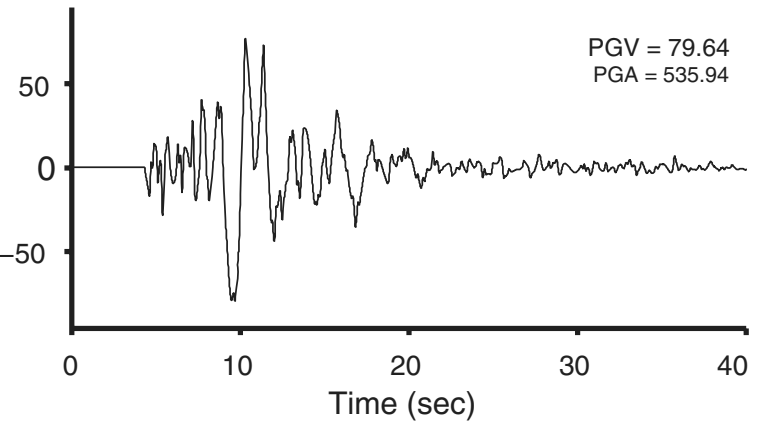

(f)

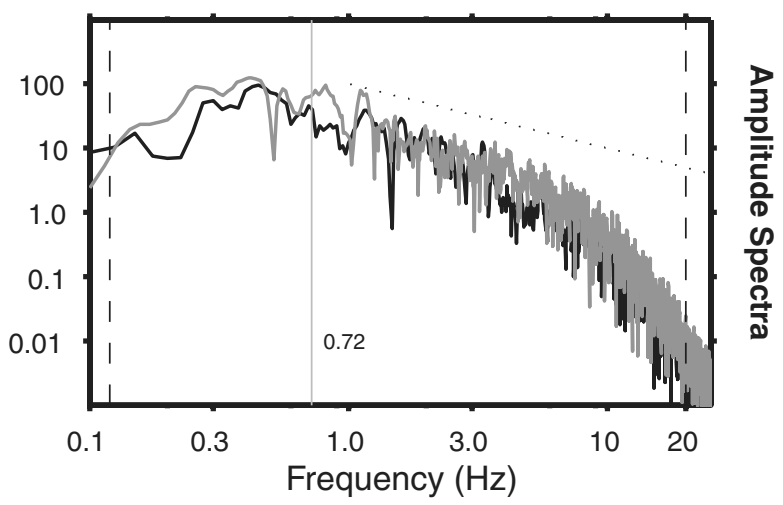

(h)

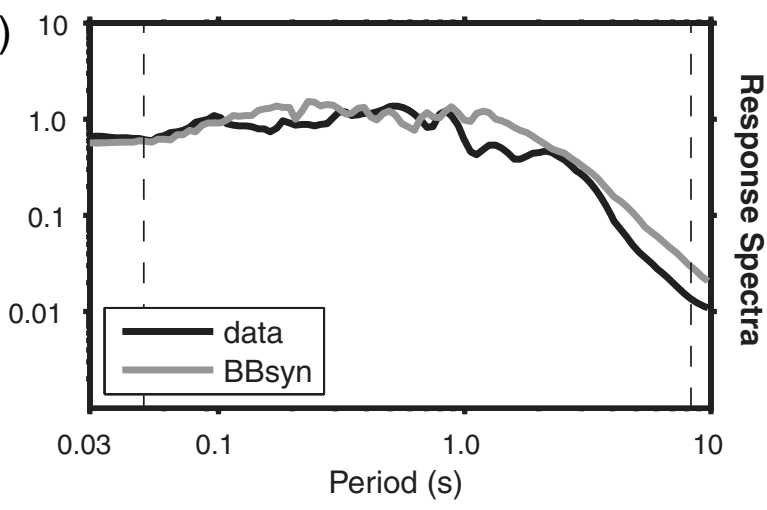

Figure 5. Comparison of east-west (left column) and north-south (right column) component synthetics (using the Wald model) and recorded ground motions at station SYLM for the 1994 Northridge earthquake. Data are shown in panels (a) and (b), hybrid broadband simulations in (c) and (d). Because absolute timing is not available for these records, the data were shifted in time to facilitate visual waveform comparison. Fourier amplitude spectra [panels (e) and (f): dotted vertical line, optimum matching frequency; broken lines, frequency range of data validity] decay approximately as $1 / f$ up to $10 \mathrm{~Hz}$ (indicated by the sloping dotted line) and show overall consistency between data and simulations. Response spectra $[\zeta=5 \%$ damping, panels $(\mathrm{g})$ and $(\mathrm{h})]$, as well as PGA and PGV values, suggest unbiased simulated ground motions. (PGV values have been computed from the waveforms shown; PGA values are obtained from the corresponding acceleration time series that are calculated by differentiating the displayed waveforms). This site (National Earthquake Hazards Reduction Program soil class C) is in the up-dip direction of the fault and experiences significant directivity effects at low frequency, indicated by strong velocity pulses in both the data and the simulations. 
East-West Component $(\mathrm{cm} / \mathrm{s})$

(a)

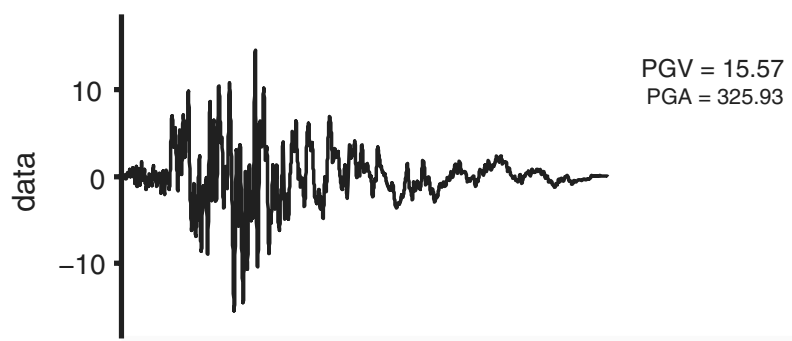

(c)

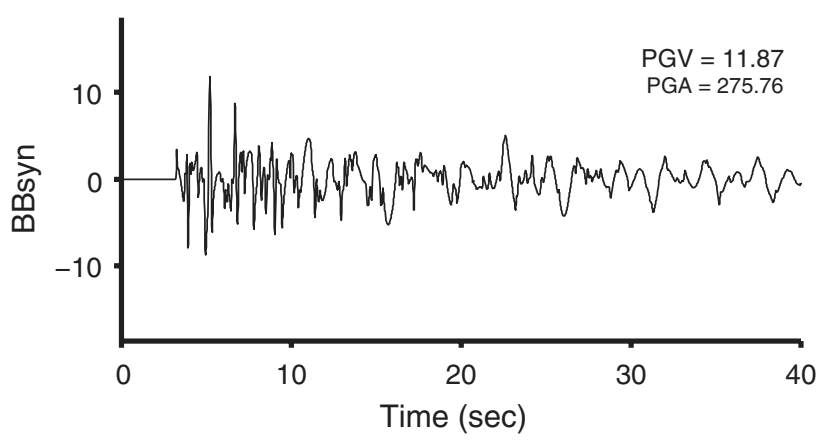

(e)

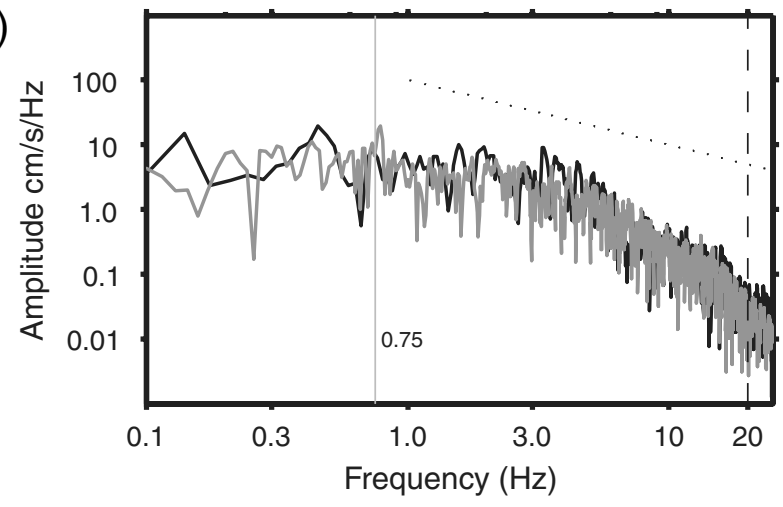

(g)

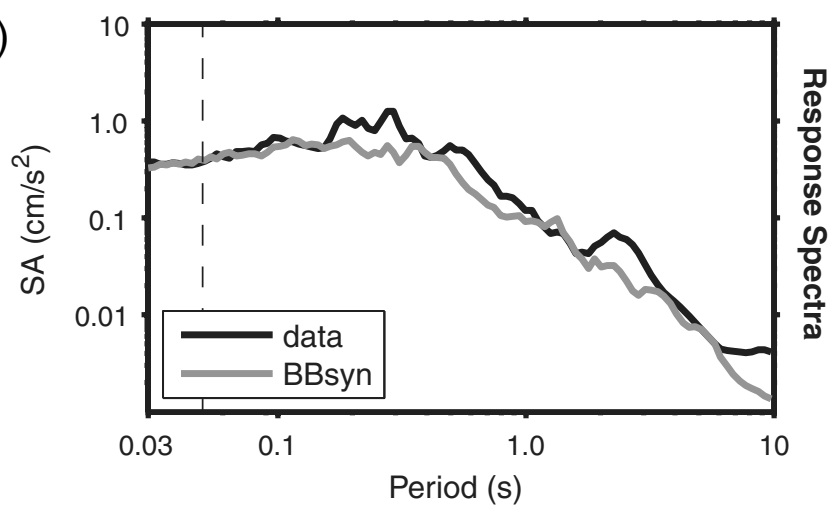

North-South Component $(\mathrm{cm} / \mathrm{s})$

(b)

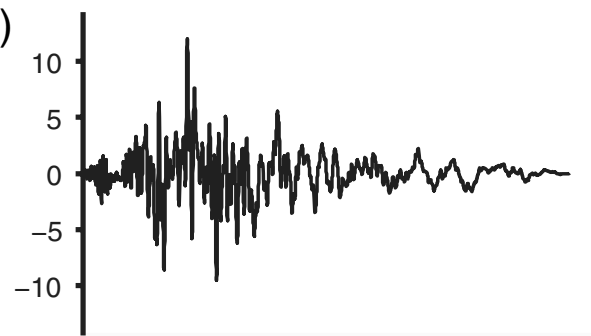

$P G V=12.03$

$P G A=189.48$

(d)
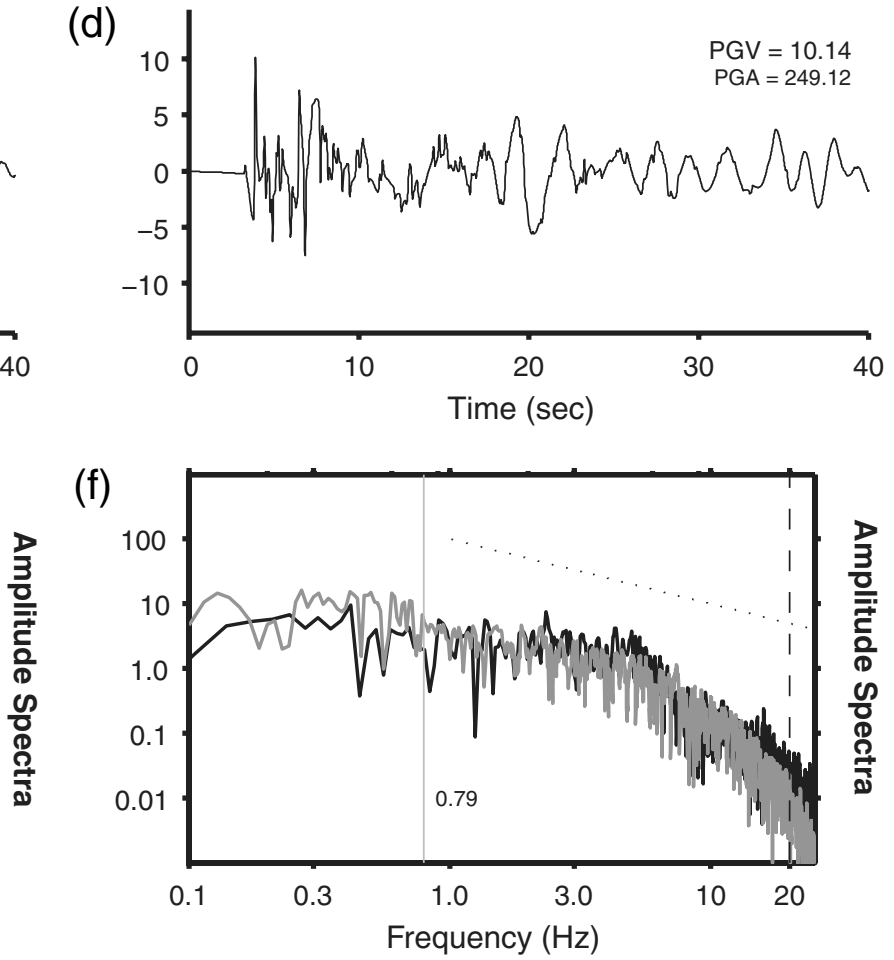

(h)

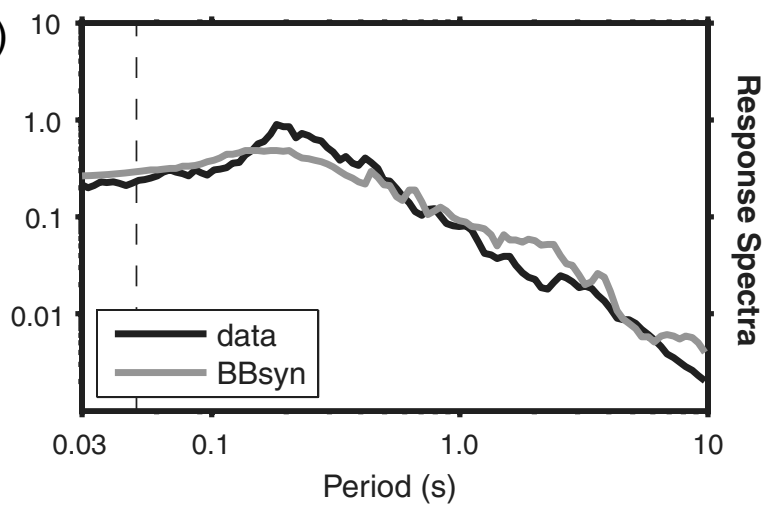

Figure 6. Same as Figure 5, for station TPFS, classified as a National Earthquake Hazards Reduction Program soil class C site. TPFS is located in the backward-directivity direction on the footwall side of the fault; note the difference in signal character to the forward-directivity site SYLM (Fig. 5).

$(f<0.05 \mathrm{~Hz})$, while, conversely, at short distances $(R \leq$ $10 \mathrm{~km}$ ), the far-field dominates already at frequencies above $f \approx 0.5 \mathrm{~Hz}$.

The 1994 Northridge earthquake occurred as a blindthrust M 6.7 buried rupture on a $40^{\circ}$ dipping fault whose rupture plane extends from about 21-km depth to 5-km depth (Fig. 3 and Fig. 4). Its hypocenter has been located at 17.5-km depth. For the selection of stations (Table 1), the smallest hypocentral distance $R_{\text {hyp }}$ is about $18 \mathrm{~km}$ (stations CNPK and SATI), whereas the shortest distance $\left(R_{\mathrm{flt}}\right)$ to the fault 
is considerably smaller $(\approx 5 \mathrm{~km})$. However, in terms of seismic radiation, $R_{\mathrm{flt}}$ is not a very useful distance metric because the seismic moment release close to rupture-plane edges is generally very low. A more meaningful distance metric in this context is the distance to the high-slip patches; however, these carry substantial uncertainty due to the differences between the rupture models and the general difficulty of precisely locating regions of high slip (due to the non-uniqueness of earthquake source inversions). We thus estimate the distance of each station to the shallow and deep slip patch, the latter being very close to the hypocenter, and find that the minimum distance of any of the 24 selected stations to any region of high moment release is on the order of 10-12 km (stations CNPK, SVSC, JENG). Given these distances to either regions of high moment release or the hypocenter, and taking into account the near-field/far-field criteria discussed previously, we conclude that for all 24 sites under consideration far-field radiation dominates for frequencies above $f \approx 0.5 \mathrm{~Hz}$, while near-field effects are important for $f<0.5 \mathrm{~Hz}$. Thus, our 3D finitedifference simulations accurately represent all near-field and intermediate-field terms for these 24 sites, capturing also directivity effects if present. Consequently, it is also justified to assume that the seismic wave field above $f \approx 0.5 \mathrm{~Hz}$ can be appropriately modeled assuming far-field radiation.

Based on the preceding discussion, we place the origin of the single-source HF (i.e., far-field) scattered wave field at the hypocenter, instead of distributing numerous scattering sources over the fault plane, which are then summed. Near-field terms for frequencies below $f \approx 0.5 \mathrm{~Hz}$ are fully captured in the LF simulations, which also include all directivity effects. As mentioned previously, directivity starts vanishing at $f \approx 1 \mathrm{~Hz}$ and hence is not present in the $\mathrm{HF}$ scattered wave field. However, it is important to note that this line of reasoning applies for the particular source-station geometry and the particular earthquake rupture model used in this study. For larger earthquakes with high-slip patches close to the Earth's surface and recording stations close to the fault, finite-fault effects should be included in the computation of the scattered wave field (see companion paper by Mena et al., 2010 for details).

\section{Scattering Parameters and Site Kappa $(\kappa)$}

In the Calculation of Site-Specific Scattering Operators section, we discussed generic choices for the scattering parameters and site kappa. Because the purpose of this study is not to solve for the scattering parameters and site kappa that best fit a given recording but instead to present a new general approach for hybrid broadband ground-motion simulations, we did not attempt to define optimal scattering parameters. We therefore only tested a small range for $\eta_{i}$ and $\eta_{s}$, identical for all stations, until we achieved a satisfactory agreement between data and simulations. The corresponding values, $\eta_{i}=0.028$ and $\eta_{s}=0.01$, remain unchanged in subsequent calculations. A thorough parameter study about the effects of various choices of $\eta_{i}$ and $\eta_{s}$ on several ground- motion parameters is reported in the companion paper by Mena et al. (2010).

Regarding site kappa, we initially chose a fixed value, $\kappa=0.03$, for all sites that was close to a standard value for generic rock sites $(\kappa=0.035-0.04)$, as defined by Boore and Joyner (1997). Additionally, we tested whether choosing site-specific kappa values would result in much better agreement between the simulations and the observations. We thus estimated $\kappa$ for all sites using the following procedure. Using strong-motion data at 133 sites, we first deamplified the original recordings using the site-amplification factors of Borcherdt (2002). The resulting amplitude spectra then appear as if they had been recorded under site conditions used in our simulations (i.e., $V S_{\min }=1000 \mathrm{~m} / \mathrm{s}$ ). These deamplified recordings are then used as input LF seismograms to generate hybrid broadband synthetics. In so doing, we generate broadband seismograms for a suite of $\kappa$ values in the range $0.01 \leq \kappa \leq 0.1$. All these simulations were then compared to the original data in terms of their spectral fall-off; for each station, the $\kappa$ value that returned the most similar spectral decay between recordings and synthetics was selected. The final $\kappa$ value was obtained by averaging over the two horizontal components.

Figure 7 displays a contour plot of the $\kappa$ values obtained by the procedure described previously in this article. Values range from $\kappa=0.01$ to $\kappa=0.085$ (for some basin sites), with a mean value of $\kappa=0.267$. The $\kappa$ values show little correlation with the reported $V_{S 30}$ or distance. In the following section, we compute hybrid broadband ground-motions for both a constant $\kappa=0.03$ and the site-specific $\kappa$. While the differences may be appreciable for amplitude spectra at certain

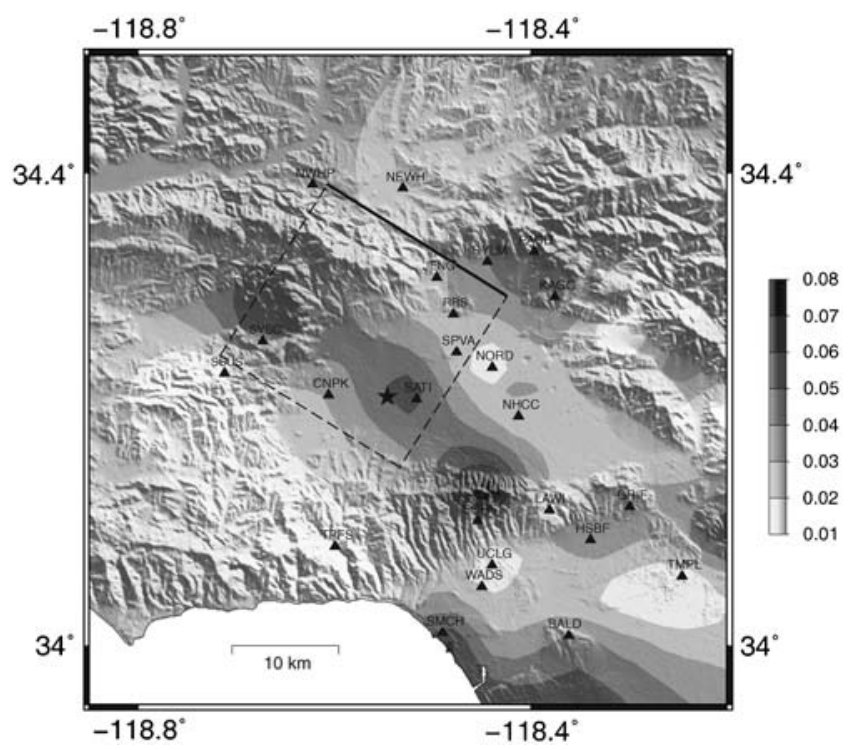

Figure 7. Map showing a contour plot of $\kappa$ values estimated using 133 sites that recorded the 1994 Northridge earthquake. Values range from $\kappa=0.010$ to $\kappa=0.085$, with a mean value of $\kappa=0.0267$. $\kappa$ values for the 24 sites of interest are reported in Table 1. 
sites, they decrease when considering the response spectral values. Once we average over the two horizontal components of motions, and subsequently over all sites, the influence of different site $\kappa$ values becomes negligible. We thus report only simulation results for uniform site $\kappa$ to also facilitate comparisons with other forward-modeling studies without access to past-event data for estimating site $\kappa$.

\section{Single-Site Ground-Motion Comparison}

To assess the accuracy of our simulations with respect to observations, we examine velocity time series, scalar groundmotion parameters (PGV and PGA), spectral-amplitude characteristics and response-spectral properties $(\zeta=5 \%$ damping).

As examples, we show comparisons at stations SYLM and TPFS (Fig. 5 and Fig. 6; see Fig. 2 for station location), both classified as National Earthquake Hazards Reduction Program (NEHRP) class C sites. Note that any comparison in terms of amplitude spectra and response spectral values needs to consider the frequency range between $f_{\min }$ and $f_{\max }$ (Table 1) in which the strong-motion data are found to be valid. Station SYLM is located in the forward-directivity direction of the rupture, while station TPFS is located in the backward-directivity direction. At station SYLM, our simulations reproduce the pulselike character of the velocity seismograms, also matching the PGV values of the two horizontal components and the PGA values to within about $20 \%$. Note that the PGA deviations depend more strongly on the particular realization of the scattering Green's function than the PGV or spectral acceleration, $\mathrm{SA}_{T}$, at longer periods (see also Mena et al., 2010).

Examining the Fourier amplitude spectra for the observed and simulated ground motions (Fig. 5e-f), we find good correspondence over almost the entire frequency range, with minor deviations for frequencies less than about $0.2 \mathrm{~Hz}$ (due to preprocessing applied to strong-motion data; Table 1). The spectral-amplitude decrease of the velocity spectra follows the expected $f^{-1}$ decay for frequencies up to $\approx 10 \mathrm{~Hz}$ and then decays more rapidly due to the relative smoothness of the applied STF. The comparison of response spectra for observed and synthetic seismograms (Fig. 5g-h) also exhibit very good agreement. The spectral response of the simulated waveforms closely follows the observations and shows only minor overprediction or underprediction.

At site TPFS (Fig. 5), located in the backward-directivity direction, ground-motion amplitudes are strongly reduced with respect to the forward-directivity site SYLM. Our simulations show good agreement with the observations in terms of PGV and PGA values (Fig. 6a-d), amplitude spectral behavior (Fig. 6e-f), and response spectral properties (Fig. 6g-h). However, deviations in spectral accelerations between data and simulations exist in both directions (i.e., underprediction and overprediction), illustrating the need for a statistical analysis that includes all 24 sites (see subsequent section). However, considering only sites SYLM and TFPS , simulated response spectra appear to be mostly unbiased with respect to the data.

Additionally, we test the potential effects of site amplification. Where indicated, we compute the empirical siteamplification factor as $F=\left(\frac{V_{\text {site }}}{V_{\text {ref }}}\right)^{m}$, where $V_{\text {site }}$ and $V_{\text {ref }}$ are the site-specific velocities and the reference shear-wave velocities, respectively, and $m$ is an empirically derived factor. Based on the methodology of Graves and Pitarka (2004), the response-spectral amplification factors of Borcherdt (2002) are then applied in the Fourier-domain to scale the amplitude spectrum; inverse fast Fourier transform then yields the scaled time series accounting for site amplification (alternatively, one could use the amplification function of Campbell and Bozorgnia, 2008). This approach allows for converting response spectra from one site condition to another. The reference condition in this study is the lowest shear-wave speed in the LF FD calculations; $V_{s}=V_{\text {ref }}=$ $1000 \mathrm{~m} / \mathrm{s}$; and the site-specific $V_{S 30}$ values are listed in Table 1. Given the range of minimum shear-wave velocities and considering typical PGA input values from our simulations, the amplification factors generally vary between 1.5 and 3.0.

\section{Ground-Motion Comparisons for All Sites Jointly}

Comparisons between simulated and observed PGV and PGA values, computed for the Wald model at all sites, are displayed in Figure 8. To jointly consider the two horizontal components of motions, we compute the geometric mean $G\left(Y_{\mathrm{ew}}, Y_{\mathrm{ns}}\right)=\sqrt{Y_{\mathrm{ew}} \cdot Y_{\mathrm{ns}}}$, where $Y$ is the ground-motion intensity measure (PGA, PGV, or $\mathrm{SA}_{T}$ ) and subscripts ew and ns denote the east-west and the north-south components, respectively.

The distance-decay of PGA and PGV of the broadband synthetics follows the trend of the data but includes both underprediction and overprediction on both components of motion for the given distance range (Fig. 8). This variation is due to the randomly generated HF-scattering contributions because the final scatterograms exhibit various waveform shapes and peak amplitudes, independent for each site and each component, resulting in considerable variability in the broadband motions (see also Mena et al., 2010). Such variability is neither uncommon nor unexpected, owing to the inherent randomness of the scattering process that contributes to the aleatory uncertainty in ground-motion prediction. This type of ground-motion behavior is also observed when comparing ground-motions for recent well-recorded earthquakes (e.g., the 1999 Chi-Chi or 2004 Parkfield events) with empirical ground-motion prediction equations (Mai, 2009).

Ensemble statistics of ground-motion simulations are further analyzed in terms of the model bias and standard error for $\mathrm{SA}_{T}$ at various periods $T_{i}$, considering all 24 stations jointly. The residual $r_{j}$ between observed and simulated SAs at each station $j$ is given as 

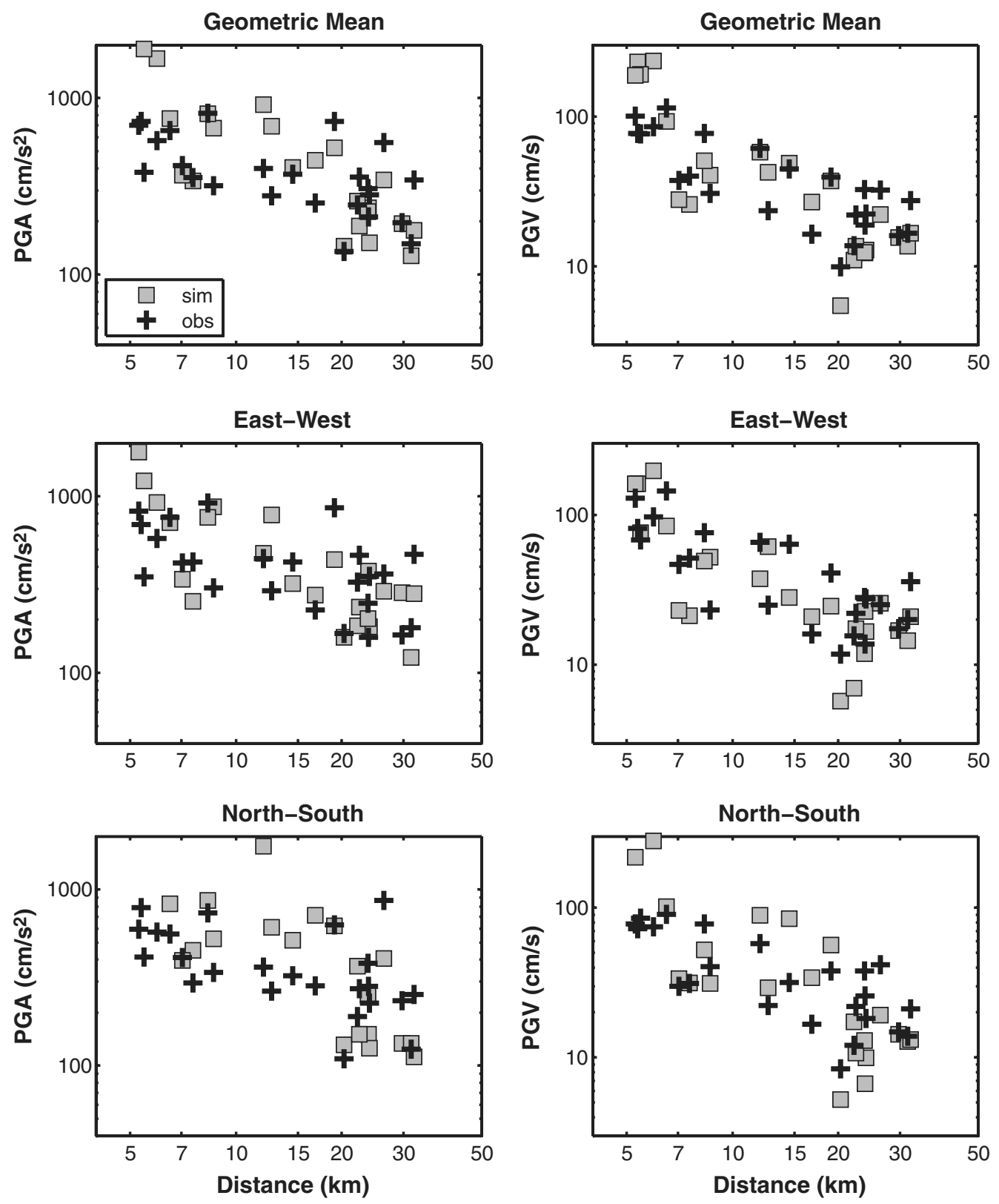

Figure 8. Comparison of recorded and simulated peak ground accelerations (left) and peak-ground velocities (right) for the Northridge earthquake at 24 sites (Table 1); distance is closest distance to the fault, $R_{\mathrm{flt}}$ ). Synthetic ground motions are computed using the Wald model and site-amplification factors by Borcherdt (2002). In general, we achieve good agreement between simulated and recorded motions, although discrepancies at individual stations exist.

$$
r_{j}\left(T_{i}\right)=\ln \left(\frac{\mathrm{SA}_{\mathrm{obs}, j}\left(T_{i}\right)}{\mathrm{SA}_{\mathrm{sim}, j}\left(T_{i}\right)}\right),
$$

where the subscripts obs and sim denote observation and simulation, respectively. The model bias $B\left(T_{i}\right)$ and standard error $E\left(T_{i}\right)$ for each period $T_{i}$ are computed by summing over all stations:

$$
B\left(T_{i}\right)=\frac{1}{N} \sum_{j=1}^{N} r_{j}\left(T_{i}\right)
$$

$$
E\left(T_{i}\right)=\left[\frac{1}{N} \sum_{j=1}^{N}\left[r_{j}\left(T_{i}\right)-B\left(T_{i}\right)\right]^{2}\right]^{1 / 2} .
$$

Thus, overprediction is given by a negative model bias and underprediction by a positive one. The $90 \%$-confidence intervals (for $N=24$ samples) are given by the following statistical function

$$
P_{90}\left(T_{i}\right)=\frac{1.714}{\sqrt{N-1}} \times E\left(T_{i}\right)
$$


where the factor 1.714 arises from the one-sided Student's t-distribution for $N-1$ degrees of freedom. The median model bias, 90\%-confidence intervals and $1-\sigma$ bounds are displayed in Figures 9-12 to help assess the uncertainties in the predictions.

Examining Figure 9 (Wald model; constant $\kappa=0.03$, site amplification applied using Borcherdt, 2002), the zero-bias level remains within the $\pm 1 \sigma$ interval; the median and the $90 \%$ confidence intervals slightly deviate from the zero-bias in the period range $1.5-4.0 \mathrm{~s}$. The $90 \%$ confidence intervals ( $1 \sigma$ intervals) occupy a range of about $\pm 0.2( \pm 0.7)$ in natural-log units, leading to a variability of a factor of $\approx 1.2(\approx 1.8)$ for observed and simulated spectral accelerations at all stations. The median spectral-acceleration value for all sites oscillates around the zero-bias line with a variation of \pm 0.2 , indicating an essentially unbiased median with about $20 \%$ variability.

Additional comparisons of broadband synthetics to data are shown in Figures 10, 11, and 12 to illustrate the effect of two source-rupture models as well as site-amplification factors. Comparing the Wald-model broadband simulations, including site-amplification (Fig. 9), to the ones without site amplification (Fig. 10), it is evident that the site-amplification factor helps to stabilize the model bias in
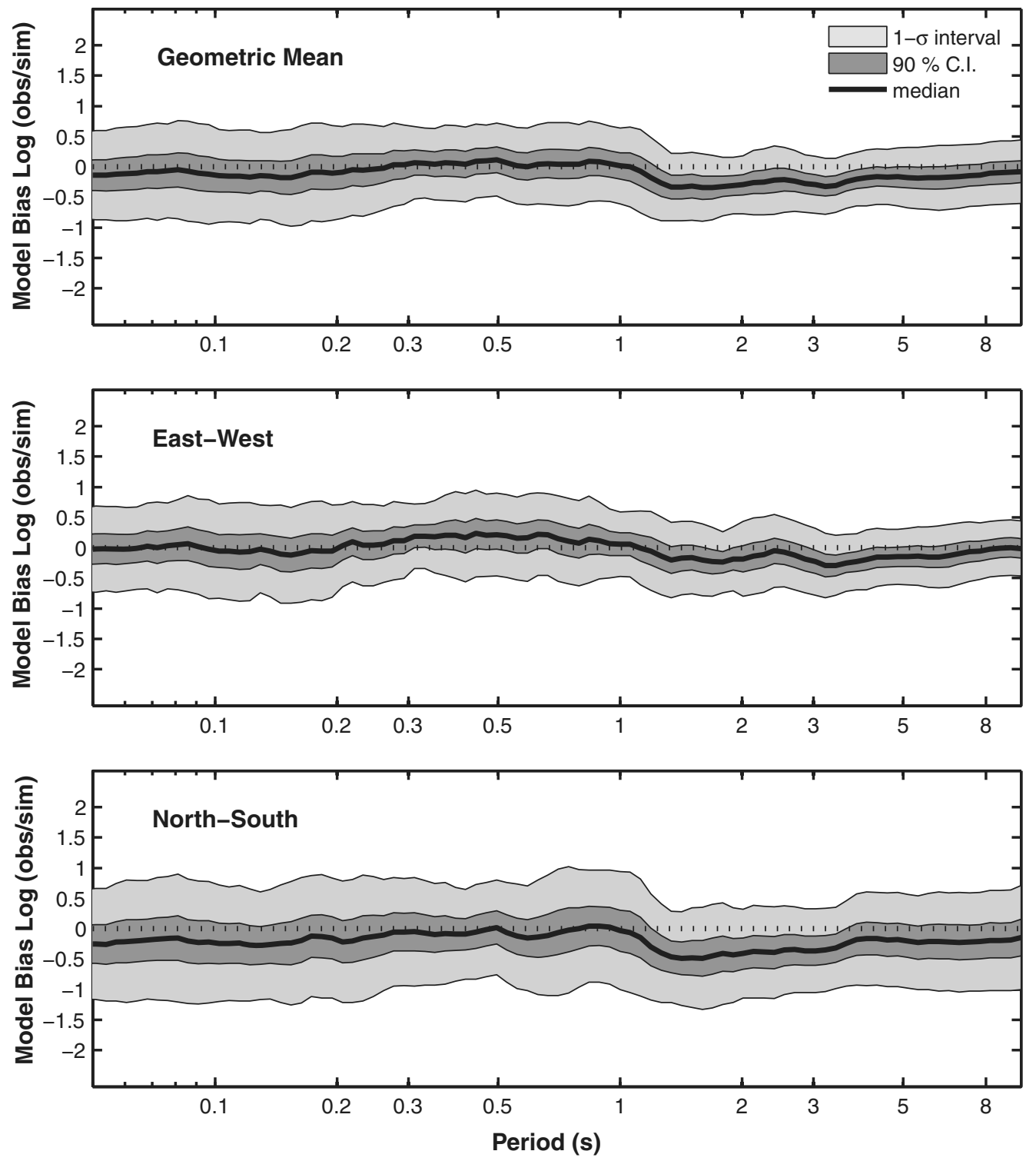

Figure 9. Model bias, computed as $\log (\mathrm{obs} / \mathrm{sim})$ in spectral acceleration, for 24 sites considered in this study; the synthetics included site-amplification factors (Borcherdt, 2002). A perfect match with the data would be indicated by zero model bias (horizontal dotted line), negative model bias indicates overprediction, and positive model bias indicates underprediction. In our simulations (for the Wald-model and for constant $\kappa=0.03 \mathrm{~s}$ ), the median fluctuates weakly around the zero-bias line. The width of the $90 \%$-confidence interval (C.I.) changes from 0.5 at short periods to 0.3 at long periods, while the $1-\sigma$ bounds vary between 1.4 and 1 . 

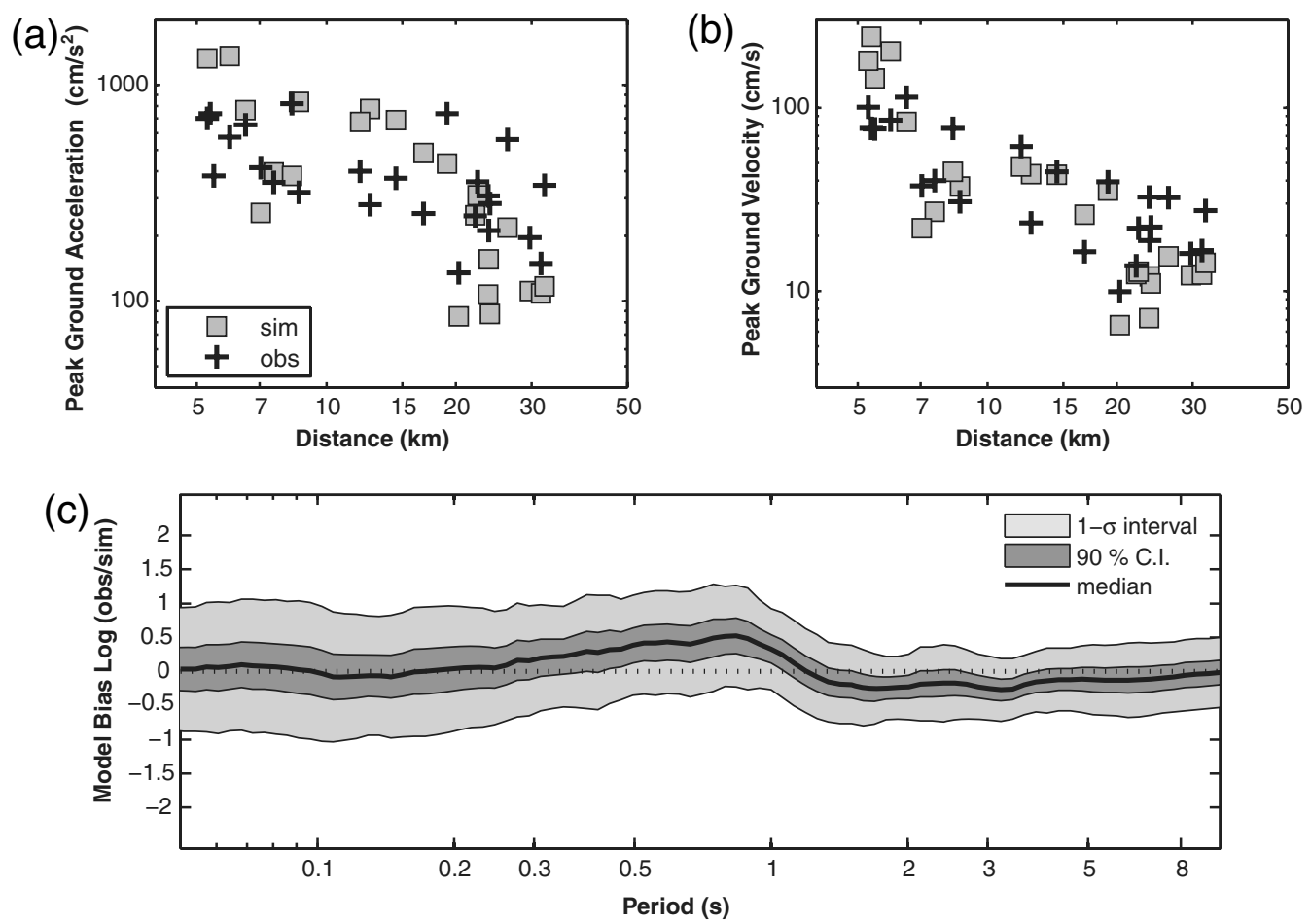

Figure 10. Ground-motion comparison of the geometric mean of the two horizontal components of (a) the recorded and simulated peak ground acceleration, (b) peak-ground velocity, and (c) spectral-acceleration model bias, using the Wald model but omitting site-amplification factors. Note the increased $1-\sigma$ range and wider $90 \%$ confidence limits, compared to Figure 9 (top panel), as well as the kink at $f \approx 1 \mathrm{~Hz}$.
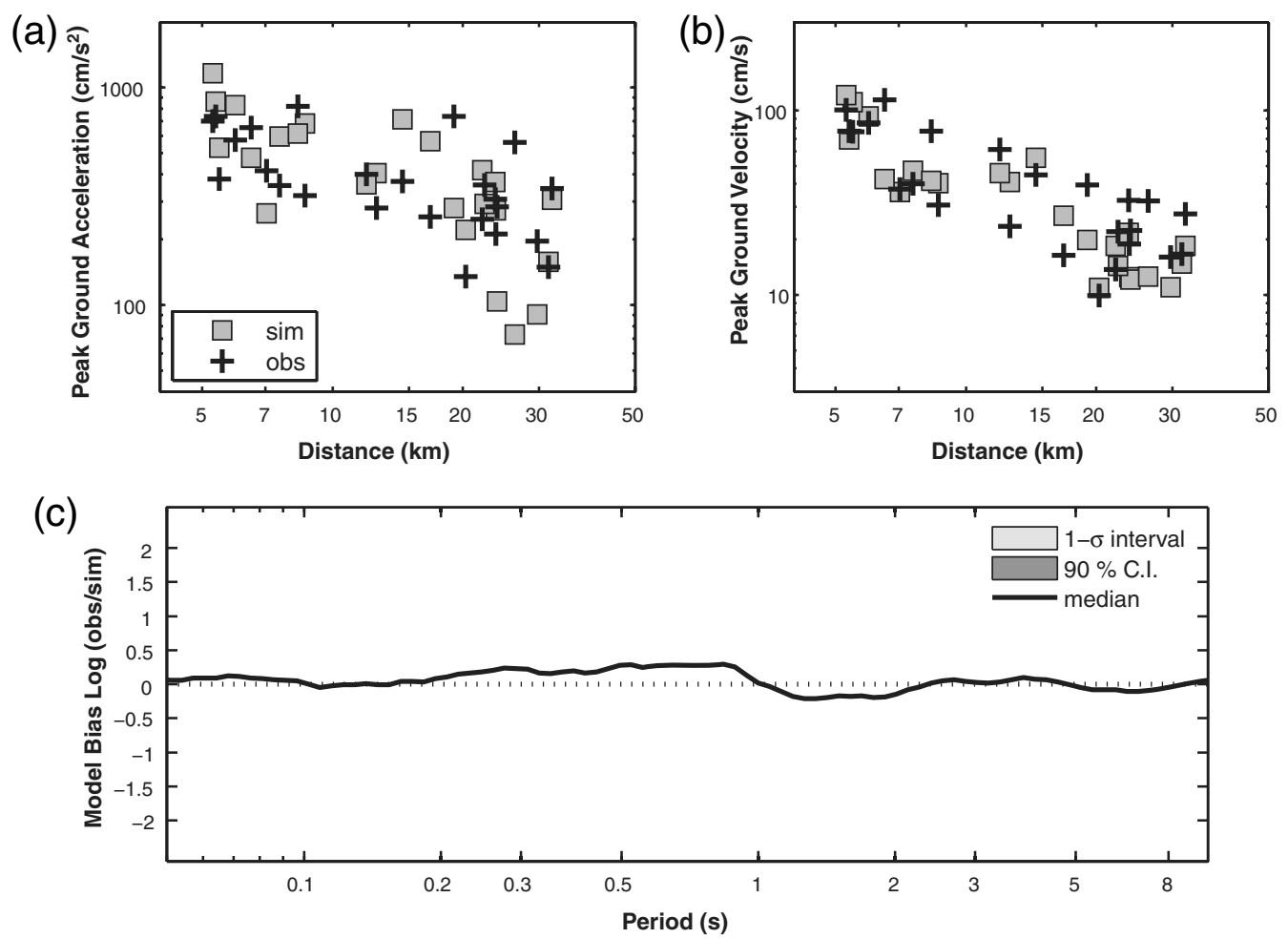

Figure 11. Ground-motion comparison of the geometric mean of the two horizontal components of (a) the recorded and simulated peak-ground acceleration, (b) peak-ground velocity, and (c) spectral-acceleration model bias, computed with the Hartzell model (Fig. 4). Site-amplification factors are included; for further details, see Figure 8 and main text. 

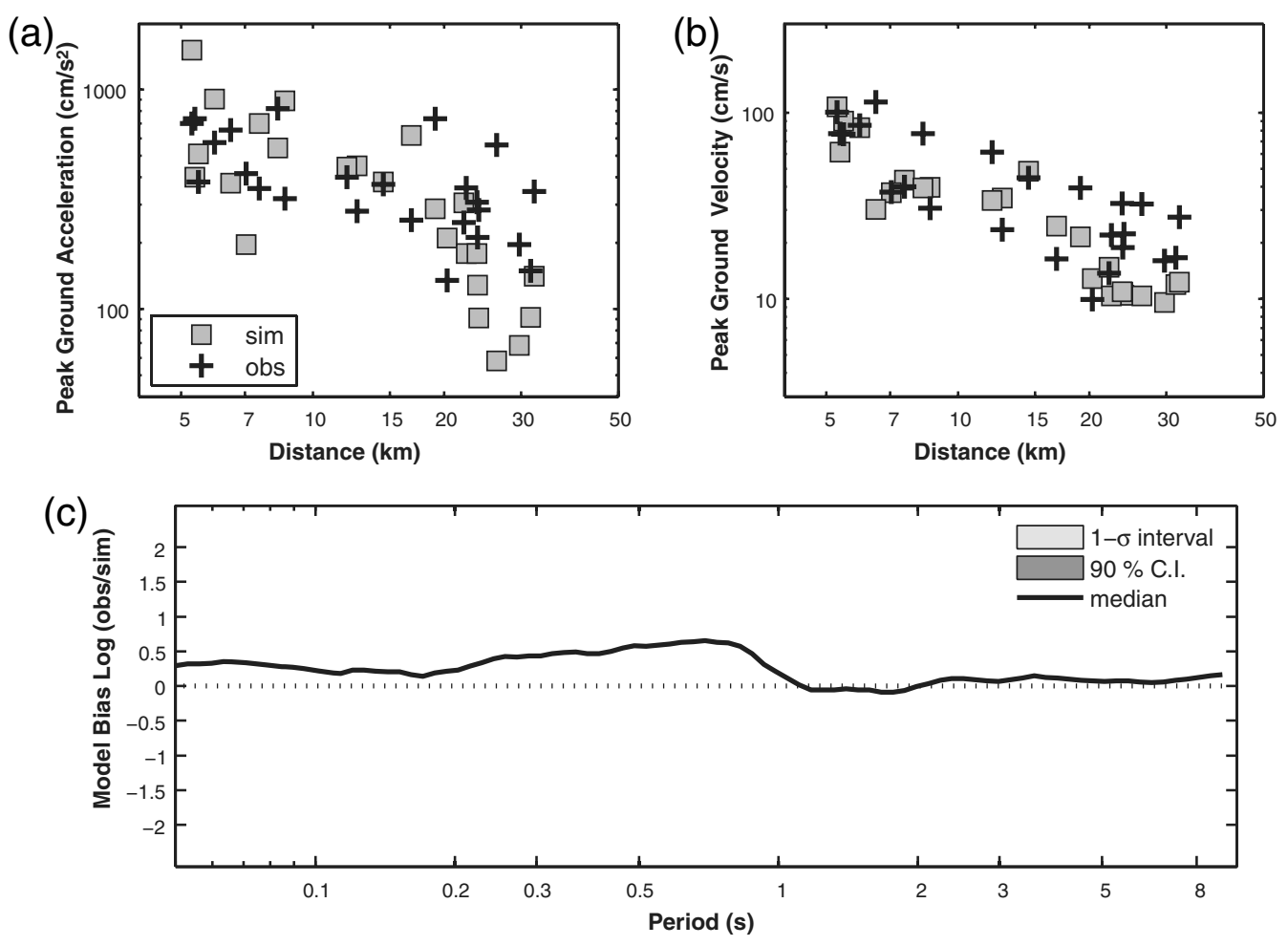

Figure 12. Same as Figure 11 but excluding site-amplification factors. Note the underprediction of spectral acceleration in the period range $0.3 \leq T \leq 1$, which is removed if site-amplification factors are included (Fig. 11).

the midperiod range $(0.3 \leq T \leq 1.0)$. Variation in the source model appears to be of minor importance, at least when examining the ensemble statistics of ground motions: the model bias for the Wald model (Fig. 9) and the Hartzell model (Fig. 11) are also similar when considering the simulations without site-amplification factors (Fig. 10 and 12). Thus, the overall ground-motion variability in our simulations is not strongly influenced by the differences in the source models but rather by the high-frequency scattering contributions.

\section{Conclusions}

Broadband groundmotions are needed to reliably estimate the potential seismic shaking in future earthquakes. In particular in the near-field regime of large earthquakes, where strong-motion databases are still sparsely populated, simulations may provide the best tool to obtain such ground-motion estimates. Deterministic techniques for wave-field computations at low frequencies (up to about $1 \mathrm{~Hz}$ ) in 3D Earth structures are well established, accounting also for the complexity in the earthquake rupture process. In contrast, methods for high-frequency ( $f \approx 1-10 \mathrm{~Hz}$ ) ground-motion simulations are largely based on empirical-stochastic approaches and generally do not consider the physics of seismic scattering due to small-scale heterogeneous Earth structure or include 3D wave-propagation effects. In this study, we present a new methodology that advances broadband ground-motion simulation by combining low-frequency 3D finite-difference synthetics with a high-frequency scattered wave-field accounting for the physics of generating incoherent seismic arrivals due to multiple $S$-to- $S$ backscattering in uniformly random isotropic media.

Our proposed method allows for rupture mechanisms that contain spatiotemporal complexity at all scales, directly affecting the low-frequency wave field and, indirectly, the scattered-wave contributions. Thus, our approach represents a physically complete and self-consistent method for hybrid broadband ground-motion simulation. Comparison with observations from the $\mathbf{M} 6.7$ Northridge earthquake shows that our approach is able to provide unbiased ground-motion predictions over the frequency range of engineering interest, satisfactorily reproducing PGA, PGV, Fourier amplitude spectra, and response spectra. This is obtained by considering LFs computed from two independent source descriptions of the Northridge earthquake, derived from Wald et al. (1996), and Hartzell et al. (1996). Our least biased results are obtained after applying frequency-dependent site-amplification factors to the broadband synthetics.

Thus, our results suggest that the proposed method can be applied for ground-motion simulations for earthquake engineering purposes, as well as for simulation-based seismic hazard assessment.

In the current implementation, we have made a number of simplifications that serve as departure for future work. While the scattering parameters $\eta_{i}$ and $\eta_{s}$ used to compute the scattering Green's function were constant for all sites, these parameters could be site specific if corresponding 
information exists. Furthermore, this study assumes that the scattering Green's function originates from a point source at the hypocenter. This approach is reasonable and very efficient for small-to-moderate size earthquakes and for all cases in which the station distances are large enough such that they can be considered far-field sites for frequencies at which the dominant seismic radiation occurs. For larger events, during which slip may come close to the Earth's surface and recording sites may be close to the fault (and potentially to a region of high moment release), the far-field approximation becomes inadequate. In this case, the computation of the high-frequency scattered wave field should include finitefault effects by summing the contributions from scattering Green's functions at individual node points of the rupture model. The companion study (Mena et al., 2010) considers such cases for very large earthquakes, based on M 6.7 TeraShake scenarios, and develops an algorithm to incorporate the scattering contribution from zones of large slip on the fault.

\section{Data and Resources}

The strong-motion recordings were provided by Robert Graves, as part of the "BB-Platform" Project of the Southern California Earthquake Center (SCEC). Site-classification information is based on the Next Generation Attenuation NGA model database of the Pacific Earthquake Engineering Research center (http://peer.berkeley.edu/nga). The computer code developed in this paper is available upon request.

\section{Acknowledgments}

We thank R. Graves and A. Pitarka for fruitful discussions; R. Graves also provided programming advice and guidance in the statistical analysis. We are indebted to Y. Zeng, who kindly supplied his scattering code that constitutes an integral part of our methodology. Comments of two anonymous reviewers and associate editor of Bulletin of the Seismological Society of America, A. Pitarka, greatly helped to improve the paper. This work was supported by the Southern California Earthquake Center (SCEC), stimulated by the SCEC Broadband Ground-Motion Simulation Project. SCEC is funded through National Science Foundation Cooperative Agreement Number EAR-0106924 and U. S. Geological Survey Cooperative Agreement Number 02HQAG0008. This is SCEC Contribution Number 1199.

\section{References}

Abrahamson, N. N. (2006). Seismic hazard assessment: Problems with current practice and future developments, First European Conference on Earthquake Engineering and Seismology Geneva, Switzerland, 3-8 September 2006.

Aki, K. (1992). Scattering conversion $P$ to $S$ versus $S$ to $P$, Bull. Seismol. Soc. Am. 82, 1969-1972.

Allmann, B. P., and P. M. Shearer (2009). Global variations of stress drop for moderate to large earthquakes, J. Geophys. Res. 114, no. B01310, doi 10.1029/2008JB005821.

Atkinson, G., and W. Silva (1997). Empirical source spectra for California earthquakes, Bull. Seismol. Soc. Am. 87, 97-113.

Bazzurro, P., B. Sjoberg, and N. Luco (2004). Post-elastic response of structures to synthetic ground motions, Report for Pacific Earthquake
Engineering Research (PEER) Center Lifelines Program Project, pp. $65-112$.

Beresnev, I., and G. Atkinson (1997). Modeling finite-fault radiation from the $\omega^{n}$ spectrum, Bull. Seismol. Soc. Am. 87, 67-84.

Berge, C., J.-C. Gariel, and P. Bernard (1998). A very broadband stochastic source model used for near source strong motion prediction, Geophys. Res. Lett. 25, no. 7, 1063-1066.

Boatwright, J., G. L. Choy, and L. C. Seekins (2002). Regional estimates of radiated seismic energy, Bull. Seismol. Soc. Am. 92, 1241-1255.

Boore, D. (1983). Stochastic simulation of high frequency ground motions based on seismological models of the radiated spectra, Bull. Seismol. Soc. Am. 73, 1865-1894.

Boore, D. M., and B. Joyner (1997). Site amplifications for generic rock sites, Bull. Seismol. Soc. Am. 87, no. 2, 327-341.

Borcherdt, R. D. (2002). Empirical evidence for acceleration-dependent amplification factors, Bull. Seismol. Soc. Am. 92, 761-782.

Campbell, K. W., and Y. Bozorgnia (2008). NGA ground motion model for the geometric mean horizontal component of PGA, PGV, PGD and 5\% damped linear elastic response spectra for periods ranging from 0.01 to 10 s, Earthq. Spectra 24, 139-171.

Day, S. M. (1998). Efficient simulation of constant $Q$ using coarse-grained memory variables, Bull. Seismol. Soc. Am. 88, 1051-1062.

Day, S. M., and C. Bradley (2001). Memory-efficient simulation of anelastic wave propagation, Bull. Seismol. Soc. Am. 91, 520-531.

Day, S. M., R. W. Graves, J. Bielak, D. Dreger, S. Larsen, K. B. Olsen, A. Pitarka, and L. Ramirez-Guzman (2008). Model for basin effects on long-period response spectra in southern California, Earthq. Spectra 24, 257-277.

Gottschämmer, E., and K. B. Olsen (2001). Accuracy of the explicit planar free- surface boundary condition implemented in a fourth-order staggered-grid velocity-stress finite-difference scheme, Bull. Seismol. Soc. Am. 91, 617-623.

Graves, R., and A. Pitarka (2004). Broadband time history simulation using a hybrid approach, paper No. 1098, World Conference on Earthquake Engineering, Vancouver (BC), Canada, August 1-6, 2004.

Graves, R., and A. Pitarka (2010). Broadband ground motion simulation using a hybrid approach, Bull. Seismol. Soc. Am., 100, no. 5A, 2095-2123.

Gritz, A. (2009). Effect of heterogeneous prestress on near-field rupture directivity, Master of Science, San Diego State University, San Diego, California, 67 pp.

Guatteri, M., P. M. Mai, and G. C. Beroza (2004). A pseudo-dynamic approximation to dynamic rupture models for strong ground motion prediction, Bull. Seismol. Soc. Am. 94, 2051-2063.

Hartzell, S., M. Guatteri, P. M. Mai, P. Liu, and M. Frisk (2005). Calculation of broadband time histories of ground motion, Part II: Kinematic and dynamic modeling using theoretical Green's functions and comparison with the 1994 Northridge earthquake, Bull. Seismol. Soc. Am. 95, 614-645.

Hartzell, S., S. Harmsen, A. Frankel, and S. Larsen (1999). Calculation of broadband time histories of ground motion: Comparison of methods and validation using strong-ground motion from the 1994 Northridge earthquake, Bull. Seismol. Soc. Am. 89, 1484-1504.

Hartzell, S., P. Liu, and C. Mendoza (1996). The 1994 Northridge, California, earthquake; investigation of rupture velocity, rise time, and highfrequency radiation, J. Geophys. Res. 101, no. 9, 20,091-20,108.

Hole, J. A. (1992). Non-linear high resolution three-dimensional seismic travel time tomography, J. Geophys. Res. 97, 6553-6562.

Hough, S. E., and J. G. Anderson (1988). High-frequency spectra observed at Anza, California-Implications for $Q$-structure, Bull. Seismol. Soc. Am. 78, 692-707.

Ichinose, G., P. Goldstein, and A. J. Rodgers (2000). Relative importance of near-, intermediate, and far-field displacement terms in layered Earth synthetic seismograms. Bull. Seismol. Soc. Am. 90, 531-536.

Irikura, K. (1986). Prediction of strong acceleration motions using empirical Greens function, Proc. 7th Japan Earthquake Engineering Symposium, Tokyo, Japan, 10-12 Dec. 1986, 151-156. 
Irikura, K., and K. Kamae (1994). Estimation of strong ground motion in broad-frequency band based on a seismic source scaling model and an empirical Green's function technique, Ann. Geofis. 37, 1721-1743.

Kamae, K., K. Irikura, and A. Pitarka (1998). A technique for simulating strong ground motion using hybrid Green's function, Bull. Seismol. Soc. Am. 88, 357-367.

Kohler, M., H. Magistrale, and R. Clayton (2003). Mantle heterogeneities and the SCEC three-dimensional seismic velocity model version 3, Bull. Seismol. Soc. Am. 93, 757-774.

Kramer, S. L. (1996).Geotechnical Earthquake Engineering, Prentice Hall, Upper Saddle River, New Jersey, 653 pp.

Liu, H. L., and D. V. Helmberger (1985). The 23:19 aftershock of the 15 October 1979 Imperial Valley earthquake: More evidence for an asperity. Bull. Seismol. Soc. Am. 75, 689-708.

Liu, P., R. J. Archuleta, and S. H. Hartzell (2006). Prediction of broadband ground-motion time histories: Hybrid low/high-frequency method with correlated random source parameters, Bull. Seismol. Soc. Am. 96, 2118-2130, doi 10.1785/0120060036.

Mai, P. M. (2009). Ground-motion complexity and scaling in the near-field of earthquake ruptures, Encyclopedia of Complexity and System Sciences, R. Meyers (Editor), Springer, ISBN 978-0-387-69572-3, pp 4435-4474.

Mai, P. M., and G. C. Beroza (2003). A hybrid method for calculating near-source, broadband seismograms: Application to strong motion prediction, Phys. Earth Planet. In. 137, no. 1-4, 183-199.

Marcinkovich, C., and K. B. Olsen (2003). On the implementation of perfectly matched layers in a 3D fourth-order velocity-stress finitedifference scheme, J. Geophys. Res., 108, 2276, doi 10.1029/ 2002JB002235.

Mayeda, K., S. Koyanagi, M. Hoshiba, K. Aki, and Y. Zeng (1992). A comparative study of scattering intrinsic and coda $Q^{-1}$ for Hawaii, Long Valley, and central California between 1.5 and $15 \mathrm{~Hz}$, J. Geophys. Res. 97, no. B5, 6643-6659.

Mena, B., P. M. Mai, K. B. Olsen, M. Purvance, and J. Brune (2010). Hybrid broadband ground-motion simulation using scattering Green's functions: Application to large-magnitude events, Bull. Seismol. Soc. Am., 100, no. 5A, 2143-2162.

Olsen, K. B. (1994). Simulation of three-dimensional wave propagation in the Salt Lake basin, Ph.D. Thesis, University of Utah, Salt Lake City, Utah, $157 \mathrm{p}$.

Olsen, K. B., S. M. Day, and C. R. Bradley (2003). Estimation of $Q$ for longperiod ( $<2 \mathrm{~s})$ waves in the Los Angeles basin, Bull. Seismol. Soc. Am. 93, 627-638.

Pulido, N., and T. Kubo(2004). Near-fault strong-motion complexity of the 2000 Tottori earthquake (Japan) from a broadband asperity model, Tectonophysics 390, 177-192.

Ripperger, J., and P. M. Mai (2004). Fast computation of static stress changes on 2D faults from final slip distributions, Geophys. Res. Lett. 31, no. 18, L18610 doi 10.1029/2004GL020594.

Raoof, M., R. B. Herrmann, and L. Malagnini (1999). Attenuation and excitation of three-component ground motion in southern California, Bull. Seismol. Soc. Am. 89, no. 4, 888-902.

Sato, (1977). Single isotropic scattering mode including wave conversionSimple theoretical model of the short-period body-wave propagation, J. Phys. Earth 25, 163-176.

Sato, H., and M. Fehler (1998). Seismic Wave Propagation and Scattering in the Heterogeneous Earth, Press AIP/Springer Verlag, New York, $308 \mathrm{pp}$.
Satoh, T. (2002). Empirical frequency-dependent radiation pattern of the 1998 Miyagiken-Nanbu earthquake in Japan, Bull. Seismol. Soc. Am. 92, no. 3, 1032-1039.

Sørensen, M., N. Pulido, and K. Atakan (2007). Sensitivity of groundmotion simulations to earthquake source parameters: A case study for Istanbul, Turkey. Bull. Seismol. Soc. Am. 97, no. 3, 881-900.

Spudich, P., and B. S. J. Chiou (2008). Directivity in NGA earthquake ground motions: Analysis using isochrone theory, Earthq. Spectra 24, 279-298.

Takenaka, H., Y. Mamada, and H. Futamure (2003). Near-source effect on radiation pattern of high-frequency $S$ waves: strong $S H-S V$ mixing observed from aftershocks of the 1997 northwestern Kagoshima, Japan, earthquakes, Phys. Earth Planet. In. 137, 31-43.

Thrainsson, H., A. S. Kiremidjian, and S. R. Winterstein (2000). Modeling of earthquake ground motion in the frequency domain, Report No. 134, June 2000, The John A. Blume Earthquake Engineering Center, Department of Civil and Environmental Engineering, Stanford University, Stanford, California, $271 \mathrm{pp}$.

Wald, D. J., T. H. Heaton, and K. W. Hudnut (1996). The slip history of the 1994 Northridge, California, earthquake determined from strongmotion, teleseismic, GPS, and leveling data, Bull. Seismol. Soc. Am. 86, 49-70.

Watson-Lamprey, J. A. (2007). The search for directivity, Seismol. Res. Lett. 78, 273.

Zeng, Y. H. (1993). Theory of scattered $P$ - and $S$-wave energy in a random isotropic scattering medium, Bull. Seismol. Soc. Am. 83, 1264-1276.

Zeng, Y. H., J. G. Anderson, and F. Su (1995). Subevent rake and random scattering effects in realistic strong ground motion simulation, Geophys. Res. Lett. 22, no. 1, 17-20.

Zeng, Y. H., F. Su, and K. Aki (1991). Scattered wave energy propagation in a random isotropic scattering medium-Part 1. Theory, J. Geophys. Res. 96, no. B1, 607-619.

Division of Physical Sciences and Engineering

King Abdullah University of Science and Technology

4700 King Abdullah University of Science and Technology

Thuwal 23955-6900

Kingdom of Saudi Arabia

(P.M.M.)

Institute of Geophysics, ETH Zurich

Sonneggstrasse 5

CH-8092 Zurich, Switzerland

imperatori@tomo.ig.erdw.ethz.ch

(W.I.)

Department of Geological Sciences

San Diego State University

San Diego, California 92182-1020

kbolsen@sciences.sdsu.edu

(K.B.O.)

Manuscript received 21 July 2008 